\title{
Orthogonal operator calculation of E1, M1 and E2 transition probabilities in Ti III and V IV
}

\author{
A.J.J. Raassen and P.H.M. Uylings \\ Van der Waals-Zeeman Instituut, Universiteit van Amsterdam, Valckenierstraat 65-67, 1018 XE Amsterdam, The Netherlands
}

Received June 21; accepted September 13, 1996

\begin{abstract}
Transition probabilities in the iron group spectra Ti III and V IV are obtained from an accurate description of the 8 lowest even and 4 lowest odd configurations of the atomic system. Results are compared with experiment and current databases.
\end{abstract}

Key words: atomic data - Sun: photosphere - stars: abundances

\section{Introduction}

Fortunately, there is a continuing increase and improvement of astrophysical observational possibilities, both by satellite and by ground based observatories. Technically advanced telescopes are used to search for faint objects and to detect the emitted light with highly dispersive equipment such as the Hubble Space Telescope (HST) and the International Ultraviolet Explorer (IUE). The vacuum ultra-violet region will be observed by FUSE-LYMAN at high resolution down to the Lyman continuum at $912 \AA$.

At the same time, this progress underlines the need for more and more accurate atomic and molecular transition probabilities. Most stellar spectra are affected with broad features or blends. Therefore, to investigate stellar objects in a state of the art way, transition probabilities (either from theoretical calculations or from laboratory observations) should necessarily be accurate to about $10 \%$ or better.

Because of their nuclear stability, iron group elements (especially the even- $Z$ ones without nuclear spin) are frequently encountered in stellar spectra. The lower (I and II) ionization stages can be observed in the photosphere, while higher ionization is seen in the emission lines of the chromosphere with its steep rise of ionic temperature; Atype stars constitute important examples of this.

To start our calculations of transition probabilities, we choose Titanium and Vanadium which are cases of intermediate complexity and allow for comparison with other

Send offprint requests to: A.J.J. Raassen work; for the near future, the study of iron and nickel which are of even greater astrophysical importance, is planned.

$\mathrm{Ti}$ and $\mathrm{V}$ are observed in a variety of stellar objects. In the sun, Ti figures in third place in terms of numbers of lines (Jaschek \& Jaschek 1995). Hundreds of Ti I and Ti II lines are observed in the solar photospheric spectrum (Moore et al. 1966; Biémont 1976). Ti II appears in midB-type stars, slowly increases towards F-type, and persists up to type M. Ti has been found overabundant (with respect to Fe) in extreme halo dwarfs (Magain 1989). Due to the lack of accurate transition probabilities of higher ionization, one was forced here to use the weaker Ti I lines to determine the abundance. Ti II is used to determine the radial velocity of A-type shell stars (Levato et al. 1995). Ti III is less frequently observed but has been seen in Ap stars (Bidelman 1966) as well as by the IUE in $\beta$ Orionis. (Rogerson \& Upson 1982).

Similarly, V I and V II show several hundreds of lines in the solar photospheric spectrum. Knowledge of accurate transition probabilities is therefore vital for the determination of the solar abundance of $\mathrm{V}$ and refining the chemical composition of the sun (Whaling et al. 1985). V II has been seen in late B-type stars and is also observed in A stars (Jaschek \& Jaschek 1995). In non-normal stars, V shows a behaviour similar to that of Fe and consequently is considered as "typical metal". $\mathrm{V}$ is slightly overabundant with respect to iron in globular cluster stars (Wheeler et al. 1989). V IV is seen in the balloon UV spectrum of the sun (Samain 1995).

Although the orthogonal operator method has been used for more than a decade now to describe energy levels of iron group elements, the present work is the first to calculate transition probabilities in this framework. It has been shown (Raassen \& Uylings 1996) to give reliable results for complex atoms, i.e. atoms with $Z>20$ and more than one electron outside closed shells. What are the advantages and disadvantages of the new orthogonal operator method? In Sect. 2, a short introduction of the method itself in relation to other current methods of producing $g f$ - or $A$-values is given; similarities as well as 
differences are discussed. In Sect. 3, details of the actual calculation as well as the numerical results are given.

\section{Orthogonal operators and other methods to calculate complex spectra}

\subsection{Some current methods to calculate transition probabilities}

Reliable absolute intensities can be obtained in quite a number of ways, but today's successful methods are almost exclusively based on intensive computing, often containing massive Configuration Interaction (CI) calculations. Pure ab initio calculations, like Multi-Configuration Hartree-Fock (MCHF, Froese Fischer 1978) and the analogous relativistic Multi-Configuration Dirac-Fock (MCDF, Parpia et al. 1996; Desclaux 1975) are most suited to produce a few, but accurate results. MCHF is able to include a large amount of electrostatic correlation and is therefore, like the Configuration Interaction Version 3 (CIV 3) code (Hibbert 1975), successful in the field of "lighter", i.e. $Z<20$, atoms. MCDF, on the other hand, is obviously better equipped for the heavier atoms. However, it can at the moment only handle a limited amount of electrostatic correlation, which means that fine structure is generally better described than absolute energies.

For the production of large quantities of reliable data, Cowan's RCN/RCG suite of programs (Cowan 1981) has gained widespread acceptance. Here, fitting to experimental energy levels with Slater-Condon parameters is vital to raise the accuracy, especially in complex energy structures with several d- or f-electrons outside closed shells where there are many levels of the same $J$-value in a relatively small energy interval.

The orthogonal operator method can be seen as a continuation of the Slater-Condon theory of atomic spectra. In the Slater-Condon theory, the Coulomb interaction between the electrons is described by means of the Slater F- and G-integrals, while the magnetic interaction due to the motion of the nucleus in the rest frame of an electron is translated into the well known spin-orbit interaction. These interactions, however, are not orthogonal which implies that adding another interaction to the system would influence the values of all previous interaction constants.

\subsection{Orthogonal operators}

Roughly a decade ago (Judd et al. 1982; Hansen et al. 1988a), this system was orthogonalized. As the parameters in the new operator set are as independent as possible, the fits are highly stabilized which offers the possibility to include new operators that account for small interactions like higher order or pure relativistic effects. As a result, the mean error $\sigma$ of the fit is reduced by an order of magnitude in a physically significant way.

Although the method is semi-empirical in character, ab initio calculations do constitute an important part of the procedure, especially to describe relativistic, e.g. fine structure effects. More specifically in connection with this work, two- particle (like dd, dp or ds) magnetic effects are relevant. Due to the small number of experimental levels in the $\mathrm{d}^{2}$ and dp configuration it is not possible to fit these parameters. Therefore they were fixed at their MCDF ab initio calculated values. In spite of the sometimes large number of parameters, the number of parameters actually varied is in many cases about the same as in the traditional Slater-Condon method. Even without varying its additional parameters, the orthogonal operator method still provides an improved description of complex spectra (Raassen \& Uylings 1996).

With respect to the classical Slater-Condon type calculations of transition probabilities, the differences are twofold: First, as the level positions and thereby the spacing between them are described in more detail, one may suppose that the corresponding eigenvectors are more accurate. These eigenvectors are used to transform the transition probabilities in pure SL-coupling to the real intermediate coupling scheme. Second, the transition integrals calculated by means of MCDF are corrected for the effect of core-polarization. We find that this correction, which is commonly a reduction of the transition integral, improves the agreement with absolute transition probabilities from experiment.

Of course there are difficulties as well, otherwise it would be hard to explain that not everyone involved in this field is using orthogonal operators. Compared to Cowan's programs, our procedure is less automatic. Parameter values, especially if they are meant to be fixed, have to be obtained either from extrapolation (which requires some experience with the region under study) or from ab initio calculation. Programs to translate Slater F- and Gintegrals to their orthogonal counterparts are available. We adapted the GRASP2 package (Parpia et al. 1996) to calculate two-electron magnetic effects; a request to obtain this program should be directed to Froese Fischer and the present authors. However, effective (i.e. higher order) electrostatic parameters may pose a problem if they can not be fitted directly, in which case one should apply extrapolation or second order perturbation theory (Uylings et al. 1993). Yet, we hope that this situation is temporary. Overview articles containing numerous parameter values for $\mathrm{d}^{N}$ (Hansen et al. 1988b), $\mathrm{d}^{N-1} \mathrm{~s}$ (van het Hof et al. 1991) and $\mathrm{d}^{N-1} \mathrm{p}$ (Uylings \& Raassen 1996) have been published and can be used to find starting values for the parameters. Everybody interested in working with orthogonal operators is invited to contact the authors or to visit our Internet address ftp://nucleus.phys.uva.nl in the directory pub/orth. 


\section{Transition probabilities}

\subsection{The need}

With efficient filters for cascade effects, one can accurately determine individual transition probabilities from lifetimes derived in observing the fluorescence following laser excitation. In iron group or other complex spectra with their hundreds or thousands of lines, this method is not practicable and the astrophysicist has to take recourse to Internet databases containing bulk data. However, many values available so far and collected e.g. in NIST compilations (Martin et al. 1988) and databases from NIST, Kurucz and Kelly, are accurate to about $50 \%$. Naturally, the data in these databases are not independent. Sometimes the NIST compilation contains experimental data but in many cases semi-empirical calculations are employed. It is hard to give error bars on this type of calculation, and if they are there at all, they are hard to substantiate.

In view of the above, it is necessary that more data become available to enable the experimentalist to decide on the real accuracy of the methods.

\subsection{The present case}

Our study of transition probabilities in the iron sequence using orthogonal operators is started with Ti III and V IV. These ions were chosen because the ground configuration contains two d-electrons, which means that they are the first ions with two particle (dd and dp) magnetic effects. In the III- and IV-spectra the configurations are relatively well separated in comparison with the I- and IIspectra in which the energies of the $3 \mathrm{~d}$ - and 4 s-electron are competitive. On the other hand the odd system is not yet perturbed by the open $3 \mathrm{p}$-shell, giving rise to a $3 \mathrm{p}^{5} 3 \mathrm{~d}^{3}$ configuration. This effect occurs in the VI-spectra and is probably already influencing the $\mathrm{V}$-spectra of the iron group elements.

To calculate the $\left(3 \mathrm{~d}^{2}+3 \mathrm{~d} 4 \mathrm{~s}\right) \rightarrow 3 \mathrm{~d} 4 \mathrm{p}$ transitions properly, it was necessary to take into account the interaction with neighbouring configurations. For that reason the even system was built from $\left(3 \mathrm{~d}^{2}+3 \mathrm{~d} 4 \mathrm{~s}+4 \mathrm{~s}^{2}+3 \mathrm{~d} 5 \mathrm{~s}+\right.$ $\left.3 \mathrm{~d} 6 \mathrm{~s}+3 \mathrm{~d} 4 \mathrm{~d}+3 \mathrm{~d} 5 \mathrm{~d}+4 \mathrm{p}^{2}\right)$ and the odd system from $(3 d 4 p+4 s 4 p+3 d 5 p+3 d 4 f)$. Interactions with other (farlying) configurations are taken into account by means of so-called effective operators. These sets of configurations are almost the same as the ones used by Wyart (1975). However he used the conventional method and his investigation was limited to energy values without the calculation of transition probabilities.

\subsection{Method}

After completing the fitting procedure, eigenvalues and eigenvectors are obtained from the diagonalization of the Hamiltonian. The eigenvectors yield the composition of
Table 1. Values for the electric dipole transition integrals in $\mathrm{Ti}$ III calculated by means of MCDF including core polarization

\begin{tabular}{c|rccc} 
& $3 \mathrm{~d} 4 \mathrm{p}$ & $4 \mathrm{~s} 4 \mathrm{p}$ & $3 \mathrm{~d} 5 \mathrm{p}$ & $3 \mathrm{~d} 4 \mathrm{f}$ \\
\hline $3 \mathrm{~d}^{2}$ & .999 & - & .231 & .617 \\
$3 \mathrm{~d} 4 \mathrm{~s}$ & -2.880 & .788 & .150 & - \\
$4 \mathrm{~s}^{2}$ & - & -2.700 & - & - \\
$3 \mathrm{~d} 5 \mathrm{~s}$ & 1.710 & - & -5.830 & - \\
$3 \mathrm{~d} 6 \mathrm{~s}$ & .501 & - & 3.550 & - \\
$3 \mathrm{~d} 4 \mathrm{~d}$ & -3.270 & - & 3.790 & -4.980 \\
$3 \mathrm{~d} 5 \mathrm{~d}$ & -.625 & - & -5.690 & 3.640 \\
$4 \mathrm{p}^{2}$ & .780 & -2.670 & - & -
\end{tabular}

Table 2. Values for the electric dipole transition integrals in V IV calculated by means of MCDF including core polarization

\begin{tabular}{c|rccc} 
& $3 \mathrm{~d} 4 \mathrm{p}$ & $4 \mathrm{~s} 4 \mathrm{p}$ & $3 \mathrm{~d} 5 \mathrm{p}$ & $3 \mathrm{~d} 4 \mathrm{f}$ \\
\hline $3 \mathrm{~d}^{2}$ & .699 & - & .185 & .577 \\
$3 \mathrm{~d} 4 \mathrm{~s}$ & -2.464 & .590 & .204 & - \\
$4 \mathrm{~s}^{2}$ & - & -2.343 & - & - \\
$3 \mathrm{~d} 5 \mathrm{~s}$ & 1.326 & - & -4.835 & - \\
$3 \mathrm{~d} 6 \mathrm{~s}$ & .398 & - & 2.764 & - \\
$3 \mathrm{~d} 4 \mathrm{~d}$ & -2.892 & - & 2.589 & -3.770 \\
$3 \mathrm{~d} 5 \mathrm{~d}$ & -.317 & - & -4.943 & 2.338 \\
$4 \mathrm{p}^{2}$ & .587 & -2.325 & - & -
\end{tabular}

the actual state in terms of pure SL-coupling, i.e. the SL-term fractions. They are used to transform the pure SL-transition matrix into the factual transition probabilities in intermediate coupling. The angular part of the SL-transition matrix is calculated from straightforward Racah algebra, the radial transition integral is obtained from the MCDF program (Parpia et al. 1996). The values of these integrals corrected for core polarization are given in Table 1 for Ti III and Table 2 for V IV.

\subsection{E1 results}

In the Tables 3 and 4 the $\log (g f)$ values for the $\left(3 \mathrm{~d}^{2}+\right.$ $3 \mathrm{~d} 4 \mathrm{~s}) \rightarrow 3 \mathrm{~d} 4 \mathrm{p}$ transitions are given for Ti III and V IV, respectively. This system is selected by cutting off the higher energy values of both the even and the odd system in the final printing procedure (not at an earlier stage of the calculation, as this would have influenced the results). The first column of these tables shows the wavelength obtained from the energy differences between the experimental level values. Wavelengths below $2000 \AA$ are given as vacuum wavelengths and above $2000 \AA$ as air wavelengths. The second column gives the $\log (g f)$ values followed by the $J$-value, energy value and the name of the level of the lower (even) level. The first character of the level name designates the configuration number: for the even levels "1" refers to $3 \mathrm{~d}^{2}$ and " 2 " to $3 \mathrm{~d} 4 \mathrm{~s}$; for the odd levels " 1 " refers to $3 \mathrm{~d} 4 \mathrm{p}$. An "** after the energy value indicates 
that the level is known, in which case (all cases here) the experimental level value is given. When unknown, the calculated energy value is given and used to calculate the wavelength. In the latter case, the wavelengths could deviate and one should be very cautious to use them for stellar spectroscopic assignments.

The electric dipole intensities are compared to those in Kurucz's database (Kurucz 1993) which is at present the most extended data base for transition probabilities. Although in many cases the results from Kurucz are comparable to ours, there are also a number of cases where large discrepancies (some orders of magnitude for the transition probabilities) occur. Striking examples of these differences are given in Tables 5 and 6 for transitions in the $3 \mathrm{~d} 4 \mathrm{~s} \rightarrow 3 \mathrm{~d} 4 \mathrm{p}$ and $3 \mathrm{~d}^{2} \rightarrow 3 \mathrm{~d} 4 \mathrm{p}$ transition array respectively. In these tables not only the differences between Kurucz's and our work are given but also the intensity numbers from the original analysis by Edlén \& Swensson (1975). These relative intensity numbers are based on visual estimates on a scale $0-25$ and comparable (on a different scale) to the intensity numbers given in the NBS compilation (Kelly 1987). From the tables it seems that our values fit the experimental data better.

\subsection{Forbidden lines}

Apart from allowed electric dipole (E1) transitions, many stellar objects show spectral lines due to magnetic dipole (M1) or electric quadrupole (E2) "forbidden" transitions that occur within complexes of the same parity. These spectral lines can only be observed when other means of decay, like E1 or non-radiative collisional transitions are unlikely, implying a low excitation temperature. Therefore, only forbidden lines in the ground state (even) system are relevant.

For the magnetic dipole transitions only an angular part, that depends on the $S$-, $L$ - and $J$-values of the initial and final states, exists. For the electric quadrupole transition, however, the transition probability contains, apart from the angular part, also a radial part. Just as in the case of the electric dipole transitions this radial part was calculated from the MCDF wavefunctions. In Tables 7 and 8 the radial integrals for the electric quadrupole transitions are given. The tables are given in the form of a symmetric matrix since the $3 \mathrm{~d}^{2}-3 \mathrm{~d} 4 \mathrm{~s}$ integral is the same as the $3 \mathrm{~d} 4 \mathrm{~s}-3 \mathrm{~d}^{2}$ integral (in both cases a $3 \mathrm{~d}-4 \mathrm{~s}$ transition is calculated). The table is somewhat complicated by the fact that for E2-transitions within the $3 \mathrm{~d} 4 \mathrm{~d}$ (or $3 \mathrm{~d} 5 \mathrm{~d}$ ) configurations, there are two non-zero operators, one for the $3 \mathrm{~d}-3 \mathrm{~d}$ and one for the $4 \mathrm{~d}-4 \mathrm{~d}$ ( or $5 \mathrm{~d}-5 \mathrm{~d}$ ) transition. For these cases, there are two rows in the table: the upper one gives the $3 \mathrm{~d}-3 \mathrm{~d}$ integral and the lower the $4 \mathrm{~d}-4 \mathrm{~d}$ (or $5 \mathrm{~d}-5 \mathrm{~d}$ ) transition integral.

Tables 9 and 10 give the $A$-values for the forbidden lines. Just as for the allowed lines only transitions involving $3 \mathrm{~d}^{2}-$ and $3 \mathrm{~d} 4 \mathrm{~s}$ levels are included. The listing is in wavelength order. The level with the lower $J$-value is given first in the designation of the transition.

\section{Conclusion}

Although the orthogonal operator method is known to describe energy levels of complex spectra more accurately (by an order of magnitude) than earlier methods, a similar claim for transition probabilities has yet to be proved. However, the transition probabilities of the present pilot cases Ti III and V IV sustain the comparison with the available experimental data successfully. Global agreement with the database of Kurucz (1993) has been found, while discrepancies seem to be in favour of the present calculation.

To avoid typing errors, all tables containing transition probabilities are computer processed. The only stage in which data typing by hand occurs is in the input of the energy fitting program. However, due to the low mean error $\sigma$ of the fit with orthogonal operators, the levels values are scrutinized almost automatically.

Complete results of the fits of both the odd and even energy system, as well as the corresponding complete transition arrays can be found in our database (anonymous ftp) at ftp://nucleus.phys.uva.nl in the directory pub/orth/ti3.

Acknowledgements. It is a pleasure to thank Dr. Biémont and Dr. Henrichs for fruitful discussions on the astrophysical relevance of this work.

\section{References}

Bidelman W.P., 1966, IAU Symposium 26, 229

Biémont E., 1976, A\&AS 26, 89

Cowan R.D., 1981, The Theory of Atomic Structure and Spectra. Berkeley. CA: University of California Press

Desclaux J.P., 1975, Comput. Phys. Commun. 9, 31

Edlén B., Swensson J.W., 1975, Phys. Scr. 12, 21

Froese Fischer C., 1978, The Hartree-Fock Method for Atoms. New York: Wiley

Hansen J.E., Uylings P.H.M., Raassen A.J.J., 1988a, Phys. Scr. 37,664

Hansen J.E., Raassen A.J.J., Uylings P.H.M., Lister G.M.S., 1988b, Nucl. Instrum. Methods B 31, 134

Hibbert A., 1975, Comput. Phys. Commun. 9, 141

Judd B.R., Hansen J.E., Raassen A.J.J., 1982, J. Phys. B: At. Mol. Phys. 15, 1457

Jaschek C., Jaschek M., 1995, The behaviour of Chemical Elements in Stars. Cambridge: Univ. Press

Kelly R.L., 1987, J. Phys. Chem. Ref. Data 16, Suppl. 1, URL address: http://cfa-www.harvard.edu/amp/data/stats/kelly.html

Kurucz R.L., 1993, Phys. Scr. T47, 110, URL address: http://cfa-www.harvard.edu/amp/data/stats/kurucz.html

Levato H., Malaroda S., Jaschek C., Jaschek M., 1995, A\&A 299, 163L

Magain P., 1989, A\&A 209, 211 
Martin G.A., Fuhr J.R., Wiese W.L., 1988, J. Phys. Chem. Ref. Dat. 17, Suppl. 3, URL address: http://aeldata.phy.nist.gov/ nist_atomic_spectra.html Moore C.E., Minnaert M.G.J., Houtgast J., 1966, The Solar Spectrum $2935 \AA$ to $8770 \AA$, NBS Monograph 61

Parpia F.A., Froese Fischer C., Grant I.P., 1996, Comput. Phys. Commun. 94, 249

Raassen A.J.J., Uylings P.H.M., 1996, Phys. Scr. T65, 84

Rogerson J.B., Upson W.L., 1982, ApJS 49, 353

Samain D. 1995 A\&AS 113, 237
Uylings P.H.M., Raassen A.J.J., Wyart J.-F., 1993, J. Phys. B: At. Mol. Opt. Phys. 26, 4683

Uylings P.H.M., Raassen A.J.J., 1996, Phys. Scr. 54, 505

van het Hof G.J., Raassen A.J.J., Uylings P.H.M., 1991, Phys. Scr. 44, 343

Whaling W., Hannaford P., Lowe R.M., Biémont E., Grevesse N., 1985, A\&A 153, 109

Wheeler C., Sneden C., Truran J.W., 1989, ARA\&A 27, 279

Wyart J.-F., 1975, Phys. Scr. 12, 33 
Table 3. Calculated $\log (g f)$ values for the $\left(3 \mathrm{~d}^{2}+3 \mathrm{~d} 4 \mathrm{~s}\right)-3 \mathrm{~d} 4 \mathrm{p}$ transition array of Ti III

\begin{tabular}{|c|c|c|c|c|c|c|c|}
\hline$\lambda(\AA)$ & $\log (g f)$ & $J_{\mathrm{f}}$ & $E_{\mathrm{f}}\left(\mathrm{cm}^{-1}\right)$ & even & $J_{\mathrm{i}}$ & $E_{\mathrm{i}}\left(\mathrm{cm}^{-1}\right)$ & odd \\
\hline 2984.744 & .152 & 2.0 & $41704.27^{*}$ & $\left.\left.2\right|_{1} ^{2} \mathrm{D}\right)^{1} \mathrm{D}$ & 2.0 & $75198.21^{*}$ & $\left.1\right|^{2} \mathrm{D}^{1} \mathrm{D}$ \\
\hline 2832.352 & -3.584 & 2.0 & $41704.27^{*}$ & $\left.\left.2\right|_{1} ^{2} \mathrm{D}\right)^{1} \mathrm{D}$ & 1.0 & $77000.23^{*}$ & $\left.1\right|^{2} D^{3} \mathrm{D}$ \\
\hline 2818.998 & -1.620 & 2.0 & $41704.27^{*}$ & $\left.\left.2\right|_{1} ^{2} \mathrm{D}\right)^{1} \mathrm{D}$ & 2.0 & $77167.43^{*}$ & $\left.1\right|^{2} \mathrm{D}^{3} \mathrm{D}$ \\
\hline 2798.916 & -1.770 & 2.0 & $41704.27^{*}$ & $\left.\left.2\right|_{1} ^{2} \mathrm{D}\right)^{1} \mathrm{D}$ & 2.0 & $77421.86^{*}$ & $\left.1\right|^{2} \mathrm{D}^{3} \mathrm{~F}$ \\
\hline 2798.713 & -2.527 & 2.0 & $41704.27^{*}$ & $\left.\left.2\right|_{1} ^{2} \mathrm{D}\right)^{1} \mathrm{D}$ & 3.0 & $77424.45^{*}$ & $\left.1\right|^{2} \mathrm{D}^{3} \mathrm{D}$ \\
\hline 2773.709 & -2.406 & 2.0 & $41704.27^{*}$ & $\left.\left.2\right|_{1} ^{2} \mathrm{D}\right)^{1} \mathrm{D}$ & 3.0 & $77746.44^{*}$ & $\left.1\right|^{2} \mathrm{D}^{3} \mathrm{~F}$ \\
\hline 2718.639 & -3.533 & 3.0 & $38425.99^{*}$ & $\left.\left.2\right|_{1} ^{2} \mathrm{D}\right)^{3} \mathrm{D}$ & 2.0 & $75198.21^{*}$ & $\left.1\right|^{2} \mathrm{D}^{1} \mathrm{D}$ \\
\hline 2701.955 & -1.466 & 2.0 & $38198.95^{*}$ & $\left.\left.2\right|_{1} ^{2} \mathrm{D}\right)^{3} \mathrm{D}$ & 2.0 & $75198.21^{*}$ & $\left.1\right|^{2} \mathrm{D}^{1} \mathrm{D}$ \\
\hline 2692.161 & -1.874 & 1.0 & $38064.35^{*}$ & $\left.\left.2\right|_{1} ^{2} \mathrm{D}\right)^{3} \mathrm{D}$ & 2.0 & $75198.21^{*}$ & $\left.1\right|^{2} \mathrm{D}^{1} \mathrm{D}$ \\
\hline 2580.443 & -.526 & 3.0 & $38425.99^{*}$ & $\left.\left.2\right|_{1} ^{2} \mathrm{D}\right)^{3} \mathrm{D}$ & 2.0 & $77167.43^{*}$ & $\left.1\right|^{2} \mathrm{D}^{3} \mathrm{D}$ \\
\hline 2576.463 & -.561 & 2.0 & $38198.95^{*}$ & $\left.\left.2\right|_{1} ^{2} \mathrm{D}\right)^{3} \mathrm{D}$ & 1.0 & $77000.23^{*}$ & $\left.1\right|^{2} \mathrm{D}^{3} \mathrm{D}$ \\
\hline 2567.556 & -.137 & 1.0 & $38064.35^{*}$ & $\left.\left.2\right|_{1} ^{2} \mathrm{D}\right)^{3} \mathrm{D}$ & 1.0 & $77000.23^{*}$ & $\left.1\right|^{2} \mathrm{D}^{3} \mathrm{D}$ \\
\hline 2565.408 & .128 & 2.0 & $38198.95^{*}$ & $\left.\left.2\right|_{1} ^{2} \mathrm{D}\right)^{3} \mathrm{D}$ & 2.0 & $77167.43^{*}$ & $\left.1\right|^{2} \mathrm{D}^{3} \mathrm{D}$ \\
\hline 2563.606 & -1.904 & 3.0 & $38425.99^{*}$ & $\left.\left.2\right|_{1} ^{2} \mathrm{D}\right)^{3} \mathrm{D}$ & 2.0 & $77421.86^{*}$ & $\left.1\right|^{2} \mathrm{D}^{3} \mathrm{~F}$ \\
\hline 2563.435 & .368 & 3.0 & $38425.99^{*}$ & $\left.\left.2\right|_{1} ^{2} \mathrm{D}\right)^{3} \mathrm{D}$ & 3.0 & $77424.45^{*}$ & $\left.1\right|^{2} \mathrm{D}^{3} \mathrm{D}$ \\
\hline 2556.576 & -2.549 & 1.0 & $38064.35^{*}$ & $\left.\left.2\right|_{1} ^{2} \mathrm{D}\right)^{3} \mathrm{D}$ & 2.0 & $77167.43^{*}$ & $\left.1\right|^{2} \mathrm{D}^{3} \mathrm{D}$ \\
\hline 2548.765 & -2.279 & 2.0 & $38198.95^{*}$ & $\left.\left.2\right|_{1} ^{2} \mathrm{D}\right)^{3} \mathrm{D}$ & 2.0 & $77421.86^{*}$ & $\left.1\right|^{2} \mathrm{D}^{3} \mathrm{~F}$ \\
\hline 2548.597 & -2.336 & 2.0 & $38198.95^{*}$ & $\left.\left.2\right|_{1} ^{2} \mathrm{D}\right)^{3} \mathrm{D}$ & 3.0 & $77424.45^{*}$ & $\left.1\right|^{2} D^{3} \mathrm{D}$ \\
\hline 2547.985 & -2.611 & 2.0 & $41704.27^{*}$ & $\left.2 \mid{ }_{1}^{2} \mathrm{D}\right)^{1} \mathrm{D}$ & 1.0 & 80939.19* & $\left.1\right|^{2} \mathrm{D}^{3} \mathrm{P}$ \\
\hline 2542.459 & -2.149 & 2.0 & $41704.27^{*}$ & $\left.\left.2\right|_{1} ^{2} \mathrm{D}\right)^{1} \mathrm{D}$ & 2.0 & $81024.47^{*}$ & $\left.1\right|^{2} \mathrm{D}^{3} \mathrm{P}$ \\
\hline 2542.442 & -2.077 & 3.0 & $38425.99^{*}$ & $\left.\left.2\right|_{1} ^{2} \mathrm{D}\right)^{3} \mathrm{D}$ & 3.0 & $77746.44^{*}$ & $\left.1\right|^{2} \mathrm{D}^{3} \mathrm{~F}$ \\
\hline 2540.048 & .204 & 1.0 & $38064.35^{*}$ & $\left.\left.2\right|_{1} ^{2} \mathrm{D}\right)^{3} \mathrm{D}$ & 2.0 & $77421.86^{*}$ & $\left.1\right|^{2} \mathrm{D}^{3} \mathrm{~F}$ \\
\hline 2527.845 & .360 & 2.0 & $38198.95^{*}$ & $\left.\left.2\right|_{1} ^{2} \mathrm{D}\right)^{3} \mathrm{D}$ & 3.0 & $77746.44^{*}$ & $\left.1\right|^{2} \mathrm{D}^{3} \mathrm{~F}$ \\
\hline 2516.066 & .471 & 3.0 & $38425.99^{*}$ & $\left.\left.2\right|_{1} ^{2} \mathrm{D}\right)^{3} \mathrm{D}$ & 4.0 & $78158.61^{*}$ & $\left.1\right|^{2} \mathrm{D}^{3} \mathrm{~F}$ \\
\hline 2413.987 & .373 & 2.0 & $41704.27^{*}$ & $\left.\left.2\right|_{1} ^{2} \mathrm{D}\right)^{1} \mathrm{D}$ & 3.0 & $83116.93^{*}$ & $\left.1\right|^{2} \mathrm{D}^{1} \mathrm{~F}$ \\
\hline 2374.990 & -.016 & 2.0 & $41704.27^{*}$ & $\left.\left.2\right|_{1} ^{2} \mathrm{D}\right)^{1} \mathrm{D}$ & 1.0 & $83796.86^{*}$ & $\left.1\right|^{2} \mathrm{D}^{1} \mathrm{P}$ \\
\hline 2346.783 & .132 & 3.0 & $38425.99^{*}$ & $\left.\left.2\right|_{1} ^{2} \mathrm{D}\right)^{3} \mathrm{D}$ & 2.0 & $81024.47^{*}$ & $\left.1\right|^{2} \mathrm{D}^{3} \mathrm{P}$ \\
\hline 2338.999 & -.140 & 2.0 & $38198.95^{*}$ & $\left.\left.2\right|_{1} ^{2} \mathrm{D}\right)^{3} \mathrm{D}$ & 1.0 & 80939.19* & $\left.1\right|^{2} \mathrm{D}^{3} \mathrm{P}$ \\
\hline 2334.340 & -.540 & 2.0 & $38198.95^{*}$ & $\left.\left.2\right|_{1} ^{2} \mathrm{D}\right)^{3} \mathrm{D}$ & 2.0 & $81024.47^{*}$ & $\left.1\right|^{2} \mathrm{D}^{3} \mathrm{P}$ \\
\hline 2331.655 & -.554 & 1.0 & $38064.35^{*}$ & $\left.\left.2\right|_{1} ^{2} \mathrm{D}\right)^{3} \mathrm{D}$ & 1.0 & 80939.19* & $\left.1\right|^{2} \mathrm{D}^{3} \mathrm{P}$ \\
\hline 2331.346 & -.473 & 1.0 & $38064.35^{*}$ & $\left.\left.2\right|_{1} ^{2} \mathrm{D}\right)^{3} \mathrm{D}$ & .0 & $80944.87^{*}$ & $\left.1\right|^{2} \mathrm{D}^{3} \mathrm{P}$ \\
\hline 2327.026 & -1.678 & 1.0 & $38064.35^{*}$ & $\left.\left.2\right|_{1} ^{2} \mathrm{D}\right)^{3} \mathrm{D}$ & 2.0 & $81024.47^{*}$ & $\left.1\right|^{2} \mathrm{D}^{3} \mathrm{P}$ \\
\hline 2245.246 & -3.595 & .0 & $32475.50^{*}$ & $\left.\left.1\right|_{0} ^{1} \mathrm{~S}\right)$ & 1.0 & $77000.23^{*}$ & $\left.1\right|^{2} \mathrm{D}^{3} \mathrm{D}$ \\
\hline 2236.895 & -2.452 & 3.0 & $38425.99^{*}$ & $\left.\left.2\right|_{1} ^{2} \mathrm{D}\right)^{3} \mathrm{D}$ & 3.0 & $83116.93^{*}$ & $\left.1\right|^{2} \mathrm{D}^{1} \mathrm{~F}$ \\
\hline 2225.588 & -2.287 & 2.0 & $38198.95^{*}$ & $\left.\left.2\right|_{1} ^{2} \mathrm{D}\right)^{3} \mathrm{D}$ & 3.0 & $83116.93^{*}$ & $\left.1\right|^{2} \mathrm{D}^{1} \mathrm{~F}$ \\
\hline 2192.398 & -2.461 & 2.0 & $38198.95^{*}$ & $\left.\left.2\right|_{1} ^{2} \mathrm{D}\right)^{3} \mathrm{D}$ & 1.0 & $83796.86^{*}$ & $\left.1\right|^{2} \mathrm{D}^{1} \mathrm{P}$ \\
\hline 2185.944 & -4.026 & 1.0 & $38064.35^{*}$ & $\left.\left.2\right|_{1} ^{2} \mathrm{D}\right)^{3} \mathrm{D}$ & 1.0 & $83796.86^{*}$ & $\left.1\right|^{2} \mathrm{D}^{1} \mathrm{P}$ \\
\hline 2062.741 & -2.903 & .0 & $32475.50^{*}$ & $\left.\left.1\right|_{0} ^{1} \mathrm{~S}\right)$ & 1.0 & 80939.19* & $\left.1\right|^{2} \mathrm{D}^{3} \mathrm{P}$ \\
\hline 1948.506 & -.770 & .0 & $32475.50^{*}$ & $\left.\left.1\right|_{0} ^{1} \mathrm{~S}\right)$ & 1.0 & $83796.86^{*}$ & $\left.1\right|^{2} \mathrm{D}^{1} \mathrm{P}$ \\
\hline 1586.625 & -2.869 & 4.0 & $14397.60^{*}$ & $\left.\left.1\right|_{2} ^{1} \mathrm{G}\right)$ & 3.0 & $77424.45^{*}$ & $\left.1\right|^{2} \mathrm{D}^{3} \mathrm{D}$ \\
\hline 1578.561 & -4.381 & 4.0 & $14397.60^{*}$ & $\left.\left.1\right|_{2} ^{1} \mathrm{G}\right)$ & 3.0 & $77746.44^{*}$ & $\left.1\right|^{2} \mathrm{D}^{3} \mathrm{~F}$ \\
\hline 1568.357 & -4.507 & 4.0 & $14397.60^{*}$ & $\left.\left.1\right|_{2} ^{1} \mathrm{G}\right)$ & 4.0 & $78158.61^{*}$ & $\left.1\right|^{2} \mathrm{D}^{3} \mathrm{~F}$ \\
\hline 1550.940 & -3.225 & 2.0 & $10721.20^{*}$ & $\left.\left.1\right|_{2} ^{3} \mathrm{P}\right)$ & 2.0 & $75198.21^{*}$ & $\left.1\right|^{2} \mathrm{D}^{1} \mathrm{D}$ \\
\hline 1548.117 & -4.573 & 1.0 & $10603.60^{*}$ & $\left.\left.1\right|_{2} ^{3} \mathrm{P}\right)$ & 2.0 & $75198.21^{*}$ & $\left.1\right|^{2} \mathrm{D}^{1} \mathrm{D}$ \\
\hline 1508.773 & -2.947 & 2.0 & $10721.20^{*}$ & $\left.\left.1\right|_{2} ^{3} \mathrm{P}\right)$ & 1.0 & $77000.23^{*}$ & $\left.1\right|^{2} \mathrm{D}^{3} \mathrm{D}$ \\
\hline 1506.101 & -1.587 & 1.0 & $10603.60^{*}$ & $\left.\left.1\right|_{2} ^{3} \mathrm{P}\right)$ & 1.0 & $77000.23^{*}$ & $\left.1\right|^{2} \mathrm{D}^{3} \mathrm{D}$ \\
\hline 1504.976 & -1.718 & 2.0 & $10721.20^{*}$ & $\left.\left.1\right|_{2} ^{3} \mathrm{P}\right)$ & 2.0 & $77167.43^{*}$ & $\left.1\right|^{2} D^{3} \mathrm{D}$ \\
\hline 1504.623 & -1.375 & .0 & $10538.40^{*}$ & $\left.\left.1\right|_{2} ^{3} \mathrm{P}\right)$ & 1.0 & $77000.23^{*}$ & $\left.1\right|^{2} \mathrm{D}^{3} \mathrm{D}$ \\
\hline 1502.317 & -1.081 & 1.0 & $10603.60^{*}$ & $\left.\left.1\right|_{2} ^{3} \mathrm{P}\right)$ & 2.0 & $77167.43^{*}$ & $\left.1\right|^{2} \mathrm{D}^{3} \mathrm{D}$ \\
\hline 1499.236 & -2.596 & 2.0 & $10721.20^{*}$ & $\left.\left.1\right|_{2} ^{3} \mathrm{P}\right)$ & 2.0 & $77421.86^{*}$ & $\left.1\right|^{2} \mathrm{D}^{3} \mathrm{~F}$ \\
\hline 1499.177 & -.865 & 2.0 & $10721.20^{*}$ & $\left.\left.1\right|_{2} ^{3} \mathrm{P}\right)$ & 3.0 & $77424.45^{*}$ & $\left.1\right|^{2} \mathrm{D}^{3} \mathrm{D}$ \\
\hline 1498.695 & -.231 & 2.0 & $8473.50 *$ & $\left.\left.1\right|_{2} ^{1} \mathrm{D}\right)$ & 2.0 & $75198.21^{*}$ & $\left.1\right|^{2} \mathrm{D}^{1} \mathrm{D}$ \\
\hline 1496.597 & -1.932 & 1.0 & $10603.60^{*}$ & $\left.\left.1\right|_{2} ^{3} \mathrm{P}\right)$ & 2.0 & $77421.86^{*}$ & $\left.1\right|^{2} \mathrm{D}^{3} \mathrm{~F}$ \\
\hline 1491.975 & -1.625 & 2.0 & $10721.20^{*}$ & $\left.\left.1\right|_{2} ^{3} \mathrm{P}\right)$ & 3.0 & $77746.44^{*}$ & $\left.1\right|^{2} \mathrm{D}^{3} \mathrm{~F}$ \\
\hline 1459.285 & -4.557 & 2.0 & $8473.50^{*}$ & $\left.\left.1\right|_{2} ^{1} \mathrm{D}\right)$ & 1.0 & $77000.23^{*}$ & $\left.1\right|^{2} \mathrm{D}^{3} \mathrm{D}$ \\
\hline 1455.733 & -2.445 & 2.0 & $8473.50 *$ & $\left.\left.1\right|_{2} ^{1} \mathrm{D}\right)$ & 2.0 & $77167.43^{*}$ & $\left.1\right|^{2} \mathrm{D}^{3} \mathrm{D}$ \\
\hline
\end{tabular}


Table 3. continued

\begin{tabular}{|c|c|c|c|c|c|c|c|}
\hline$\lambda(\AA)$ & $\log (g f)$ & $\mathrm{J}_{f}$ & $\mathrm{E}_{f}\left(\mathrm{~cm}^{-1}\right)$ & even & $\mathrm{J}_{i}$ & $\mathrm{E}_{i}\left(\mathrm{~cm}^{-1}\right)$ & odd \\
\hline 1455.195 & .208 & 4.0 & $14397.60^{*}$ & $\left.\left.1\right|_{2} ^{1} \mathrm{G}\right)$ & 3.0 & $83116.93^{*}$ & $\left.1\right|^{2} \mathrm{D}^{1} \mathrm{~F}$ \\
\hline 1450.361 & -2.075 & 2.0 & $8473.50^{*}$ & $\left.\left.1\right|_{2} ^{1} \mathrm{D}\right)$ & 2.0 & $77421.86^{*}$ & $\left.1\right|^{2} D^{3} F$ \\
\hline 1450.306 & -2.890 & 2.0 & $8473.50^{*}$ & $\left.\left.1\right|_{2} ^{1} \mathrm{D}\right)$ & 3.0 & $77424.45^{*}$ & $\left.1\right|^{2} \mathrm{D}^{3} \mathrm{D}$ \\
\hline 1443.565 & -4.045 & 2.0 & $8473.50^{*}$ & $\left.\left.1\right|_{2} ^{1} \mathrm{D}\right)$ & 3.0 & $77746.44^{*}$ & $\left.1\right|^{2} \mathrm{D}^{3} \mathrm{~F}$ \\
\hline 1424.136 & -.798 & 2.0 & $10721.20 *$ & $\left.1{ }_{2}^{3} \mathrm{P}\right)$ & 1.0 & 80939.19* & $\left.1\right|^{2} \mathrm{D}^{3} \mathrm{P}$ \\
\hline 1422.409 & -.302 & 2.0 & $10721.20^{*}$ & $\left.\left.1\right|_{2} ^{3} \mathrm{P}\right)$ & 2.0 & $81024.47^{*}$ & $\left.1\right|^{2} \mathrm{D}^{3} \mathrm{P}$ \\
\hline 1421.755 & -.997 & 1.0 & $10603.60^{*}$ & $\left.\left.1\right|_{2} ^{3} \mathrm{P}\right)$ & 1.0 & 80939.19* & $\left.1\right|^{2} \mathrm{D}^{3} \mathrm{P}$ \\
\hline 1421.641 & -.883 & 1.0 & $10603.60^{*}$ & $\left.\left.1\right|_{2} ^{3} \mathrm{P}\right)$ & .0 & $80944.87^{*}$ & $\left.1\right|^{2} \mathrm{D}^{3} \mathrm{P}$ \\
\hline 1420.439 & -.901 & .0 & $10538.40 *$ & $\left.\left.1\right|_{2} ^{3} \mathrm{P}\right)$ & 1.0 & $80939.19^{*}$ & $\left.1\right|^{2} \mathrm{D}^{3} \mathrm{P}$ \\
\hline 1420.034 & -.809 & 1.0 & $10603.60 *$ & $\left.\left.1\right|_{2} ^{3} \mathrm{P}\right)$ & 2.0 & $81024.47^{*}$ & $\left.1\right|^{2} \mathrm{D}^{3} \mathrm{P}$ \\
\hline 1381.297 & -3.243 & 2.0 & $10721.20^{*}$ & $\left.\left.1\right|_{2} ^{3} \mathrm{P}\right)$ & 3.0 & $83116.93^{*}$ & $\left.1\right|^{2} \mathrm{D}^{1} \mathrm{~F}$ \\
\hline 1379.963 & -2.194 & 2.0 & $8473.50^{*}$ & $\left.\left.1\right|_{2} ^{1} \mathrm{D}\right)$ & 1.0 & 80939.19* & $\left.1\right|^{2} \mathrm{D}^{3} \mathrm{P}$ \\
\hline 1378.341 & -3.390 & 2.0 & $8473.50^{*}$ & $\left.\left.1\right|_{2} ^{1} \mathrm{D}\right)$ & 2.0 & $81024.47^{*}$ & $\left.1\right|^{2} \mathrm{D}^{3} \mathrm{P}$ \\
\hline 1368.445 & -2.232 & 2.0 & $10721.20 *$ & $\left.\left.1\right|_{2} ^{3} \mathrm{P}\right)$ & 1.0 & $83796.86^{*}$ & $\left.1\right|^{2} \mathrm{D}^{1} \mathrm{P}$ \\
\hline 1366.246 & -3.256 & 1.0 & $10603.60^{*}$ & $\left.\left.1\right|_{2} ^{3} \mathrm{P}\right)$ & 1.0 & $83796.86^{*}$ & $\left.1\right|^{2} \mathrm{D}^{1} \mathrm{P}$ \\
\hline 1365.030 & -2.811 & .0 & $10538.40^{*}$ & $\left.\left.1\right|_{2} ^{3} \mathrm{P}\right)$ & 1.0 & $83796.86^{*}$ & $\left.1\right|^{2} \mathrm{D}^{1} \mathrm{P}$ \\
\hline 1339.703 & -1.847 & 2.0 & $8473.50^{*}$ & $\left.\left.1\right|_{2} ^{1} \mathrm{D}\right)$ & 3.0 & $83116.93 *$ & $\left.1\right|^{2} \mathrm{D}^{1} \mathrm{~F}$ \\
\hline 1333.097 & -3.986 & 3.0 & $184.90^{*}$ & $\left.\left.1\right|_{2} ^{3} \mathrm{~F}\right)$ & 2.0 & $75198.21^{*}$ & $\left.1\right|^{2} D^{1} D$ \\
\hline 1329.819 & -2.083 & 2.0 & $.00 *$ & $\left.\left.1\right|_{2} ^{3} \mathrm{~F}\right)$ & 2.0 & $75198.21^{*}$ & $\left.1\right|^{2} D^{1} D$ \\
\hline 1327.609 & -.601 & 2.0 & $8473.50^{*}$ & $\left.\left.1\right|_{2} ^{1} \mathrm{D}\right)$ & 1.0 & $83796.86^{*}$ & $\left.1\right|^{2} \mathrm{D}^{1} \mathrm{P}$ \\
\hline 1298.996 & -.280 & 3.0 & $184.90^{*}$ & $\left.\left.1\right|_{2} ^{3} \mathrm{~F}\right)$ & 2.0 & $77167.43^{*}$ & $\left.1\right|^{2} D^{3} \mathrm{D}$ \\
\hline 1298.697 & -.317 & 2.0 & $.00 *$ & $\left.\left.1\right|_{2} ^{3} \mathrm{~F}\right)$ & 1.0 & $77000.23 *$ & $\left.1\right|^{2} D^{3} \mathrm{D}$ \\
\hline 1298.633 & -.121 & 4.0 & $420.40^{*}$ & $\left.\left.1\right|_{2} ^{3} \mathrm{~F}\right)$ & 3.0 & $77424.45^{*}$ & $\left.1\right|^{2} D^{3} D$ \\
\hline 1295.884 & -.680 & 2.0 & $.00 *$ & $\left.\left.1\right|_{2} ^{3} \mathrm{~F}\right)$ & 2.0 & $77167.43^{*}$ & $\left.1\right|^{2} D^{3} \mathrm{D}$ \\
\hline 1294.717 & -.653 & 3.0 & $184.90^{*}$ & $\left.\left.1\right|_{2} ^{3} \mathrm{~F}\right)$ & 2.0 & $77421.86^{*}$ & $\left.1\right|^{2} \mathrm{D}^{3} \mathrm{~F}$ \\
\hline 1294.674 & -.600 & 3.0 & $184.90^{*}$ & $\left.\left.1\right|_{2} ^{3} \mathrm{~F}\right)$ & 3.0 & $77424.45^{*}$ & $\left.1\right|^{2} D^{3} D$ \\
\hline 1293.225 & -.507 & 4.0 & $420.40^{*}$ & $\left.\left.1\right|_{2} ^{3} \mathrm{~F}\right)$ & 3.0 & $77746.44^{*}$ & $\left.1\right|^{2} \mathrm{D}^{3} \mathrm{~F}$ \\
\hline 1291.625 & -.878 & 2.0 & $.00 *$ & $\left.\left.1\right|_{2} ^{3} \mathrm{~F}\right)$ & 2.0 & $77421.86^{*}$ & $\left.1\right|^{2} D^{3} F$ \\
\hline 1291.582 & -1.874 & 2.0 & $.00 *$ & $\left.\left.1\right|_{2} ^{3} \mathrm{~F}\right)$ & 3.0 & $77424.45^{*}$ & $\left.1\right|^{2} \mathrm{D}^{3} \mathrm{D}$ \\
\hline 1289.299 & -.737 & 3.0 & $184.90^{*}$ & $\left.\left.1\right|_{2} ^{3} \mathrm{~F}\right)$ & 3.0 & $77746.44^{*}$ & $\left.1\right|^{2} \mathrm{D}^{3} \mathrm{~F}$ \\
\hline 1286.369 & -.304 & 4.0 & $420.40^{*}$ & $\left.\left.1\right|_{2} ^{3} \mathrm{~F}\right)$ & 4.0 & $78158.61^{*}$ & $\left.1\right|^{2} \mathrm{D}^{3} \mathrm{~F}$ \\
\hline 1286.233 & -1.661 & 2.0 & $.00^{*}$ & $\left.\left.1\right|_{2} ^{3} \mathrm{~F}\right)$ & 3.0 & $77746.44^{*}$ & $\left.1\right|^{2} \mathrm{D}^{3} \mathrm{~F}$ \\
\hline 1282.484 & -1.478 & 3.0 & $184.90 *$ & $\left.\left.1\right|_{2} ^{3} \mathrm{~F}\right)$ & 4.0 & $78158.61^{*}$ & $\left.1\right|^{2} D^{3} F$ \\
\hline 1237.018 & -3.070 & 3.0 & $184.90^{*}$ & $\left.\left.1\right|_{2} ^{3} \mathrm{~F}\right)$ & 2.0 & $81024.47^{*}$ & $\left.1\right|^{2} \mathrm{D}^{3} \mathrm{P}$ \\
\hline 1235.495 & -3.341 & 2.0 & $.00^{*}$ & $\left.\left.1\right|_{2} ^{3} \mathrm{~F}\right)$ & 1.0 & 80939.19* & $\left.1\right|^{2} \mathrm{D}^{3} \mathrm{P}$ \\
\hline 1234.195 & -3.910 & 2.0 & $.00^{*}$ & $\left.\left.1\right|_{2} ^{3} \mathrm{~F}\right)$ & 2.0 & $81024.47^{*}$ & $\left.1\right|^{2} \mathrm{D}^{3} \mathrm{P}$ \\
\hline 1209.241 & -3.196 & 4.0 & $420.40^{*}$ & $\left.\left.1\right|_{2} ^{3} \mathrm{~F}\right)$ & 3.0 & $83116.93^{*}$ & $\left.1\right|^{2} \mathrm{D}^{1} \mathrm{~F}$ \\
\hline 1205.807 & -3.666 & 3.0 & $184.90 *$ & $\left.\left.1\right|_{2} ^{3} \mathrm{~F}\right)$ & 3.0 & $83116.93 *$ & $\left.1\right|^{2} \mathrm{D}^{1} \mathrm{~F}$ \\
\hline 1203.124 & -4.407 & 2.0 & $.00^{*}$ & $\left.\left.1\right|_{2} ^{3} \mathrm{~F}\right)$ & 3.0 & $83116.93^{*}$ & $\left.1\right|^{2} \mathrm{D}^{1} \mathrm{~F}$ \\
\hline 1193.362 & -3.411 & 2.0 & $.00^{*}$ & $\left.\left.1\right|_{2} ^{3} \mathrm{~F}\right)$ & 1.0 & $83796.86^{*}$ & $\left.1\right|^{2} \mathrm{D}^{1} \mathrm{P}$ \\
\hline
\end{tabular}


Table 4. Calculated $\log (g f)$ values for the $\left(3 \mathrm{~d}^{2}+3 \mathrm{~d} 4 \mathrm{~s}\right)-3 d 4 p$ transition array of V IV

\begin{tabular}{|c|c|c|c|c|c|c|c|}
\hline$\lambda(\AA)$ & $\log (g f)$ & $J_{\mathrm{f}}$ & $E_{\mathrm{f}}\left(\mathrm{cm}^{-1}\right)$ & even & $J_{\mathrm{i}}$ & $E_{\mathrm{i}}\left(\mathrm{cm}^{-1}\right)$ & odd \\
\hline 268.292 & .139 & 2.0 & $100200.70^{*}$ & $\left.\left.2\right|_{1} ^{2} \mathrm{D}\right)^{1} \mathrm{D}$ & 2.0 & 144273.10* & $\left.1\right|^{2} \mathrm{D}^{1} \mathrm{D}$ \\
\hline 2177.161 & -3.837 & 2.0 & $100200.70^{*}$ & $\left.\left.2\right|_{1} ^{2} \mathrm{D}\right)^{1} \mathrm{D}$ & 1.0 & $146117.70^{*}$ & $\left.1\right|^{2} \mathrm{D}^{3} \mathrm{D}$ \\
\hline 162.484 & -1.442 & 2.0 & $100200.70^{*}$ & $\left.\left.2\right|_{1} ^{2} \mathrm{D}\right)^{1} \mathrm{D}$ & 2.0 & $146429.30^{*}$ & \\
\hline 142.745 & -2.416 & 2.0 & $100200.70^{*}$ & $\left.2 \mid{ }_{1}^{2} \mathrm{D}\right)^{1} \mathrm{D}$ & 3.0 & $146855.10^{*}$ & $\left.1\right|^{2} \mathrm{D}^{3} \mathrm{D}$ \\
\hline 2129.956 & -1.401 & 2.0 & $100200.70 *$ & $\left.2 \mid{ }_{1}^{2} \mathrm{D}\right)^{1} \mathrm{D}$ & 2.0 & $147135.20^{*}$ & $\left.1\right|^{2} \mathrm{D}^{3} \mathrm{~F}$ \\
\hline 106.556 & -1.927 & 2.0 & $100200.70^{*}$ & $\left.\left.2\right|_{1} ^{2} \mathrm{D}\right)^{1} \mathrm{D}$ & 3.0 & $147656.50^{*}$ & $\left.1\right|^{2} \mathrm{D}^{3} \mathrm{~F}$ \\
\hline 105.700 & -3.414 & 3.0 & $96798.00 *$ & $\left.\left.2\right|_{1} ^{2} \mathrm{D}\right)^{3} \mathrm{D}$ & 2.0 & $144273.10^{*}$ & $\left.1\right|^{2} \mathrm{D}^{1} \mathrm{D}$ \\
\hline 088.719 & -1.178 & 2.0 & $96412.10^{*}$ & $\left.\left.2\right|_{1} ^{2} \mathrm{D}\right)^{3} \mathrm{D}$ & 2.0 & $144273.10^{*}$ & $\left.1\right|^{2} \mathrm{D}^{1} \mathrm{D}$ \\
\hline 079.334 & -1.685 & 1.0 & 96196.10* & $\left.\left.2\right|_{1} ^{2} \mathrm{D}\right)^{3} \mathrm{D}$ & 2.0 & $144273.10^{*}$ & $\left.1\right|^{2} \mathrm{D}^{1} \mathrm{D}$ \\
\hline 2014.207 & -.513 & 3.0 & 96798.00* & $\left.2 \mid{ }_{1}^{2} \mathrm{D}\right)^{3} \mathrm{D}$ & 2.0 & $146429.30 *$ & $\left.1\right|^{2} D^{3} \mathrm{D}$ \\
\hline 011.196 & -.573 & 2.0 & $96412.10^{*}$ & $\left.\left.2\right|_{1} ^{2} \mathrm{D}\right)^{3} \mathrm{D}$ & 1.0 & $146117.70^{*}$ & $\left.1\right|^{2} \mathrm{D}^{3} \mathrm{D}$ \\
\hline 2002.492 & -.169 & 1.0 & $96196.10^{*}$ & $\left.\left.2\right|_{1} ^{2} \mathrm{D}\right)^{3} \mathrm{D}$ & 1.0 & $146117.70^{*}$ & $\left.1\right|^{2} \mathrm{D}^{3} \mathrm{D}$ \\
\hline 1999.312 & .069 & 2.0 & $96412.10^{*}$ & $\left.2 \mid{ }_{1}^{2} \mathrm{D}\right)^{3} \mathrm{D}$ & 2.0 & $146429.30^{*}$ & $\left.1\right|^{2} D^{3} \mathrm{D}$ \\
\hline 97.719 & .340 & 3.0 & 967 & $\left.2\right|_{1} ^{2} \mathrm{I}$ & 3.0 & $146855.10^{*}$ & $\left.1\right|^{2} \mathrm{D}^{3} \mathrm{D}$ \\
\hline 90.715 & -1.235 & 1.0 & $96196.10^{*}$ & $\left.2\right|_{1} ^{2} \mathrm{I}$ & 2.0 & $146429.30^{*}$ & $\left.1\right|^{2} \mathrm{D}^{3} \mathrm{D}$ \\
\hline 1986.602 & -3.257 & 3.0 & 96798.00* & $\left.2\right|_{1} ^{2} \mathrm{D}$ & 2.0 & $147135.20 *$ & $\left.1\right|^{2} \mathrm{D}^{3} \mathrm{~F}$ \\
\hline 1982.436 & -1.763 & 2.0 & 9641 & $2 \mid{ }_{1}^{2} \mathrm{D}$ & 3.0 & $146855.10^{*}$ & $\left.1\right|^{2} D^{3} \mathrm{D}$ \\
\hline 71.488 & -1.276 & 2.0 & & $\left.\left.2\right|_{1} ^{2} \mathrm{D}\right)^{3} \mathrm{D}$ & 2.0 & $147135.20^{*}$ & \\
\hline 240 & -1.931 & 3.0 & & 3 & 3.0 & 147656 . & $\left.1\right|^{2} \mathrm{D}^{3} \mathrm{~F}$ \\
\hline 29 & .162 & 1.0 & & $\left.2\right|_{1} ^{2} \mathrm{D}$ & 2.0 & 14713 & $\left.1\right|^{2} \mathrm{D}^{3} \mathrm{~F}$ \\
\hline 1952.122 & -2.792 & 2.0 & 10020 & $\left.2\right|_{1} ^{2} \mathrm{I}$ & 1.0 & $151427.00^{*}$ & \\
\hline 1951.433 & .333 & 2.0 & 964 & $\left.2\right|_{1} ^{2} \mathrm{I}$ & 3.0 & $147656.50^{*}$ & $\left.1\right|^{2} \mathrm{D}^{3} \mathrm{~F}$ \\
\hline 46.790 & -1.849 & 2.0 & 1002 & $\left.2\right|_{1} ^{2} \mathrm{I}$ & 2.0 & $151567.30^{*}$ & \\
\hline 39.067 & .449 & 3.0 & & 3 & 4.0 & $148369.20^{*}$ & $\left.1\right|^{2} \mathrm{D}^{3} \mathrm{~F}$ \\
\hline 361.573 & .351 & 2.0 & & $\left.2\right|_{1} ^{2} \mathrm{I}$ & 3.0 & & \\
\hline 1825.840 & .108 & 3.0 & & $\left.2\right|_{1} ^{2} \mathrm{I}$ & 2.0 & $151567.30^{*}$ & $\left.1\right|^{2} \mathrm{D}^{3} \mathrm{P}$ \\
\hline 1817.689 & -.164 & 2.0 & 964 & $\left.2\right|_{1} ^{2} \mathrm{I}$ & 1.0 & $151427.00^{*}$ & $\left.1\right|^{2} \mathrm{D}^{3} \mathrm{P}$ \\
\hline 3.066 & -.544 & 2.0 & 964 & $\left.2\right|_{1} ^{2} \mathrm{I}$ & 2.0 & $151567.30^{*}$ & \\
\hline 1810.581 & -.556 & 1.0 & 961 & $\left.2\right|_{1} ^{2} \mathrm{~L}$ & 1.0 & 151427.00* & $\left.1\right|^{2} \mathrm{D}^{3} \mathrm{P}$ \\
\hline 9.856 & -.491 & 1.0 & 961 & $\left.\left.2\right|_{1} ^{2} \mathrm{D}\right)^{3} \mathrm{D}$ & .0 & 15144 & \\
\hline 02 & -.031 & 2.0 & * & & 1.0 & & $\left.1\right|^{2} \mathrm{D}^{1} \mathrm{P}$ \\
\hline 1805.993 & -1.674 & 1.0 & $0^{*}$ & & 2.0 & $151567.30^{*}$ & $\left.1\right|^{2} \mathrm{D}^{3} \mathrm{P}$ \\
\hline 1750.679 & -2.185 & 3.0 & $00^{*}$ & & 3.0 & & \\
\hline 8.931 & -1.899 & 2.0 & $10^{*}$ & $\left.\left.2\right|_{1} ^{2} \mathrm{D}\right)^{3} \mathrm{D}$ & 3.0 & 153918.70* & $\left.1\right|^{2} \mathrm{D}^{1} \mathrm{~F}$ \\
\hline 1690.520 & -2.603 & 2.0 & 964 & $\left.\left.2\right|_{1} ^{2} \mathrm{D}\right)^{3} \mathrm{D}$ & 1.0 & $155565.50^{*}$ & $\left.1\right|^{2} \mathrm{D}^{1} \mathrm{P}$ \\
\hline 684.369 & -4.146 & 1.0 & $0 *$ & $\left.2 \mid{ }_{1}^{2} \mathrm{D}\right)^{3} \mathrm{D}$ & 1.0 & $155565.50^{*}$ & $\left.1\right|^{2} \mathrm{D}^{1} \mathrm{P}$ \\
\hline 964.733 & -3.446 & .0 & & & 1.0 & $146117.70^{*}$ & $\left.1\right|^{2} \mathrm{D}^{3} \mathrm{D}$ \\
\hline 917.727 & -2.845 & .0 & & & 1.0 & & \\
\hline 884.147 & -.821 & .0 & 4246 & $\left.\left.1\right|_{0} ^{1} \mathrm{~S}\right)$ & 1.0 & $155565.50^{*}$ & $\left.1\right|^{2} \mathrm{D}^{1} \mathrm{P}$ \\
\hline 778.429 & -2.589 & 4.0 & $20 *$ & $\left.1 \mid{ }_{2}^{1} \mathrm{G}\right)$ & 3.0 & $146855.10^{*}$ & $\left.1\right|^{2} D^{3} \mathrm{D}$ \\
\hline 773.603 & -5.685 & 4.0 & $0^{*}$ & $\left.1 \mid{ }_{2}^{1} \mathrm{G}\right)$ & 3.0 & $6.50 *$ & $\left.1\right|^{2} \mathrm{D}^{3} \mathrm{~F}$ \\
\hline 9.361 & -4.204 & 4.0 & & $\left.1 \mid{ }_{2}^{1} \mathrm{G}\right)$ & 4.0 & $148369.20 *$ & $\left.1\right|^{2} \mathrm{D}^{3} \mathrm{~F}$ \\
\hline & -2.844 & 2.0 & & $\left.\left.1\right|_{2} ^{3} \mathrm{P}\right)$ & 2.0 & & \\
\hline 763.161 & -4.607 & 1.0 & $132:$ & $\left.\left.1\right|_{2} ^{3} \mathrm{P}\right)$ & 2.0 & $144273.10^{*}$ & $\left.1\right|^{2} \mathrm{D}^{1} \mathrm{D}$ \\
\hline 753.810 & -3.036 & 2.0 & $30 *$ & $\left.\left.1\right|_{2} ^{3} \mathrm{P}\right)$ & 1.0 & $146117.70^{*}$ & $\left.1\right|^{2} D^{3} \mathrm{D}$ \\
\hline 752.567 & -1.614 & 1.0 & 1323 & $\left.\left.1\right|_{2} ^{3} \mathrm{P}\right)$ & 1.0 & $146117.70^{*}$ & $\left.1\right|^{2} D^{3} \mathrm{D}$ \\
\hline 752.044 & -1.727 & 2.0 & & $\left.\left.1\right|_{2} ^{3} \mathrm{P}\right)$ & 2.0 & $146429.30^{*}$ & $\left.1\right|^{2} D^{3} \mathrm{D}$ \\
\hline 751.909 & -1.371 & .0 & & $\left.1{ }_{2}^{3} \mathrm{P}\right)$ & 1.0 & & $\left.1\right|^{2} D^{3} \mathrm{D}$ \\
\hline 750.807 & -1.038 & 1.0 & $13239.20^{*}$ & $\left.\left.1\right|_{2} ^{3} \mathrm{P}\right)$ & 2.0 & $146429.30 *$ & $\left.1\right|^{2} \mathrm{D}^{3} \mathrm{D}$ \\
\hline 750.110 & -.330 & 2.0 & 1095 & $\left.1 \mid{ }_{2}^{1} \mathrm{D}\right)$ & 2.0 & $144273.10^{*}$ & $\left.1\right|^{2} \mathrm{D}^{1} \mathrm{D}$ \\
\hline 749.643 & -.843 & 2.0 & $13458.30^{*}$ & $\left.\left.1\right|_{2} ^{3} \mathrm{P}\right)$ & 3.0 & $146855.10^{*}$ & $\left.1\right|^{2} D^{3} \mathrm{D}$ \\
\hline 748.072 & -3.253 & 2.0 & $.30 *$ & $\left.\left.1\right|_{2} ^{3} \mathrm{P}\right)$ & 2.0 & $147135.20^{*}$ & $\left.1\right|^{2} \mathrm{D}^{3} \mathrm{~F}$ \\
\hline 746.848 & -2.429 & 1.0 & $13239.20^{*}$ & $\left.1{ }_{2}^{3} \mathrm{P}\right)$ & 2.0 & $147135.20^{*}$ & $\left.1\right|^{2} \mathrm{D}^{3} \mathrm{~F}$ \\
\hline 745.166 & -1.981 & 2.0 & $13458.30^{*}$ & $\left.\left.1\right|_{2} ^{3} \mathrm{P}\right)$ & 3.0 & $147656.50^{*}$ & $\left.1\right|^{2} \mathrm{D}^{3} \mathrm{~F}$ \\
\hline & -4.297 & 2.0 & & $\left.1 \mid{ }_{2}^{1} \mathrm{D}\right)$ & 1.0 & $146117.70^{*}$ & $\left.1\right|^{2} D^{3} \mathrm{D}$ \\
\hline 738.171 & -2.518 & 2.0 & $10959.30 *$ & $\left.1 \mid{ }_{2}^{1} \mathrm{D}\right)$ & 2.0 & $146429.30^{*}$ & $\left.1\right|^{2} D^{3} \mathrm{D}$ \\
\hline
\end{tabular}


Table 4. continued

\begin{tabular}{|c|c|c|c|c|c|c|c|}
\hline$\lambda(\AA)$ & $\log (\mathrm{gf})$ & $\mathrm{J}_{f}$ & $\mathrm{E}_{f}\left(\mathrm{~cm}^{-1}\right)$ & even & $\mathrm{J}_{i}$ & $\mathrm{E}_{i}\left(\mathrm{~cm}^{-1}\right)$ & odd \\
\hline 737.858 & .175 & 4.0 & $18391.20^{*}$ & $\left.\left.1\right|_{2} ^{1} \mathrm{G}\right)$ & 3.0 & 153918.70* & $\left.1\right|^{2} \mathrm{D}^{1} \mathrm{~F}$ \\
\hline 735.858 & -2.438 & 2.0 & 10959.30* & $\left.\left.1\right|_{2} ^{1} \mathrm{D}\right)$ & 3.0 & $146855.10^{*}$ & $\left.1\right|^{2} D^{3} \mathrm{D}$ \\
\hline 734.344 & -1.853 & 2.0 & 10959.30* & $\left.1 \mid{ }_{2}^{1} \mathrm{D}\right)$ & 2.0 & $147135.20^{*}$ & $\left.1\right|^{2} \mathrm{D}^{3} \mathrm{~F}$ \\
\hline 731.544 & -4.121 & 2.0 & 10959.30* & $\left.\left.1\right|_{2} ^{1} \mathrm{D}\right)$ & 3.0 & $147656.50^{*}$ & $\left.1\right|^{2} \mathrm{D}^{3} \mathrm{~F}$ \\
\hline 724.802 & -.834 & 2.0 & $13458.30^{*}$ & $\left.\left.1\right|_{2} ^{3} \mathrm{P}\right)$ & 1.0 & $151427.00 *$ & $\left.1\right|^{2} \mathrm{D}^{3} \mathrm{P}$ \\
\hline 724.066 & -.324 & 2.0 & $13458.30^{*}$ & $\left.\left.1\right|_{2} ^{3} \mathrm{P}\right)$ & 2.0 & $151567.30^{*}$ & $\left.1\right|^{2} \mathrm{D}^{3} \mathrm{P}$ \\
\hline 723.653 & -1.015 & 1.0 & $13239.20^{*}$ & $\left.\left.1\right|_{2} ^{3} \mathrm{P}\right)$ & 1.0 & $151427.00^{*}$ & $\left.1\right|^{2} \mathrm{D}^{3} \mathrm{P}$ \\
\hline 723.537 & -.905 & 1.0 & $13239.20^{*}$ & $\left.\left.1\right|_{2} ^{3} \mathrm{P}\right)$ & .0 & $151449.10^{*}$ & $\left.1\right|^{2} \mathrm{D}^{3} \mathrm{P}$ \\
\hline 723.044 & -.931 & .0 & $13122.80^{*}$ & $\left.\left.1\right|_{2} ^{3} \mathrm{P}\right)$ & 1.0 & $151427.00^{*}$ & $\left.1\right|^{2} \mathrm{D}^{3} \mathrm{P}$ \\
\hline 722.919 & -.841 & 1.0 & $13239.20^{*}$ & $\left.\left.1\right|_{2} ^{3} \mathrm{P}\right)$ & 2.0 & $151567.30^{*}$ & $\left.1\right|^{2} \mathrm{D}^{3} \mathrm{P}$ \\
\hline 711.944 & -2.701 & 2.0 & $13458.30^{*}$ & $\left.\left.1\right|_{2} ^{3} \mathrm{P}\right)$ & 3.0 & $153918.70^{*}$ & $\left.1\right|^{2} \mathrm{D}^{1} \mathrm{~F}$ \\
\hline 711.907 & -1.928 & 2.0 & 10959.30* & $\left.\left.1\right|_{2} ^{1} \mathrm{D}\right)$ & 1.0 & $151427.00 *$ & $\left.1\right|^{2} \mathrm{D}^{3} \mathrm{P}$ \\
\hline 711.197 & -2.730 & 2.0 & 10959.30* & $\left.\left.1\right|_{2} ^{1} \mathrm{D}\right)$ & 2.0 & $151567.30 *$ & $\left.1\right|^{2} \mathrm{D}^{3} \mathrm{P}$ \\
\hline 703.694 & -1.931 & 2.0 & $13458.30^{*}$ & $\left.\left.1\right|_{2} ^{3} \mathrm{P}\right)$ & 1.0 & $155565.50 *$ & $\left.1\right|^{2} \mathrm{D}^{1} \mathrm{P}$ \\
\hline 702.611 & -3.172 & 1.0 & $13239.20^{*}$ & $\left.\left.1\right|_{2} ^{3} \mathrm{P}\right)$ & 1.0 & $155565.50 *$ & $\left.1\right|^{2} \mathrm{D}^{1} \mathrm{P}$ \\
\hline 702.037 & -2.706 & .0 & $13122.80^{*}$ & $\left.\left.1\right|_{2} ^{3} \mathrm{P}\right)$ & 1.0 & $155565.50^{*}$ & $\left.1\right|^{2} \mathrm{D}^{1} \mathrm{P}$ \\
\hline 699.499 & -1.423 & 2.0 & 10959.30* & $\left.\left.1\right|_{2} ^{1} \mathrm{D}\right)$ & 3.0 & $153918.70^{*}$ & $\left.1\right|^{2} \mathrm{D}^{1} \mathrm{~F}$ \\
\hline 694.697 & -3.471 & 3.0 & $325.40^{*}$ & $\left.\left.1\right|_{2} ^{3} \mathrm{~F}\right)$ & 2.0 & $144273.10^{*}$ & $\left.1\right|^{2} \mathrm{D}^{1} \mathrm{D}$ \\
\hline 693.130 & -1.857 & 2.0 & $.00^{*}$ & $\left.\left.1\right|_{2} ^{3} \mathrm{~F}\right)$ & 2.0 & $144273.10^{*}$ & $\left.1\right|^{2} \mathrm{D}^{1} \mathrm{D}$ \\
\hline 691.533 & -.560 & 2.0 & $10959.30^{*}$ & $\left.\left.1\right|_{2} ^{1} \mathrm{D}\right)$ & 1.0 & $155565.50^{*}$ & $\left.1\right|^{2} \mathrm{D}^{1} \mathrm{P}$ \\
\hline 684.444 & -.224 & 3.0 & $325.40^{*}$ & $\left.\left.1\right|_{2} ^{3} \mathrm{~F}\right)$ & 2.0 & $146429.30^{*}$ & $\left.1\right|^{2} D^{3} \mathrm{D}$ \\
\hline 684.380 & -.334 & 2.0 & $.00^{*}$ & $\left.\left.1\right|_{2} ^{3} \mathrm{~F}\right)$ & 1.0 & $146117.70^{*}$ & $\left.1\right|^{2} \mathrm{D}^{3} \mathrm{D}$ \\
\hline 684.367 & -.075 & 4.0 & $734.70^{*}$ & $\left.\left.1\right|_{2} ^{3} \mathrm{~F}\right)$ & 3.0 & $146855.10^{*}$ & $\left.1\right|^{2} \mathrm{D}^{3} \mathrm{D}$ \\
\hline 682.923 & -.828 & 2.0 & $.00^{*}$ & $\left.\left.1\right|_{2} ^{3} \mathrm{~F}\right)$ & 2.0 & $146429.30 *$ & $\left.1\right|^{2} \mathrm{D}^{3} \mathrm{D}$ \\
\hline 682.456 & -.729 & 3.0 & $325.40^{*}$ & $\left.\left.1\right|_{2} ^{3} \mathrm{~F}\right)$ & 3.0 & $146855.10^{*}$ & $\left.1\right|^{2} D^{3} \mathrm{D}$ \\
\hline 681.153 & -.915 & 3.0 & $325.40^{*}$ & $\left.\left.1\right|_{2} ^{3} \mathrm{~F}\right)$ & 2.0 & $147135.20^{*}$ & $\left.1\right|^{2} \mathrm{D}^{3} \mathrm{~F}$ \\
\hline 680.943 & -2.062 & 2.0 & $.00^{*}$ & $\left.\left.1\right|_{2} ^{3} \mathrm{~F}\right)$ & 3.0 & $146855.10^{*}$ & $\left.1\right|^{2} \mathrm{D}^{3} \mathrm{D}$ \\
\hline 680.634 & -.732 & 4.0 & $734.70^{*}$ & $\left.\left.1\right|_{2} ^{3} \mathrm{~F}\right)$ & 3.0 & $147656.50^{*}$ & $\left.1\right|^{2} \mathrm{D}^{3} \mathrm{~F}$ \\
\hline 679.647 & -.754 & 2.0 & $.00^{*}$ & $\left.\left.1\right|_{2} ^{3} \mathrm{~F}\right)$ & 2.0 & $147135.20^{*}$ & $\left.1\right|^{2} \mathrm{D}^{3} \mathrm{~F}$ \\
\hline 678.743 & -.631 & 3.0 & $325.40^{*}$ & $\left.\left.1\right|_{2} ^{3} \mathrm{~F}\right)$ & 3.0 & $147656.50^{*}$ & $\left.1\right|^{2} \mathrm{D}^{3} \mathrm{~F}$ \\
\hline 677.348 & -.317 & 4.0 & $734.70^{*}$ & $\left.\left.1\right|_{2} ^{3} \mathrm{~F}\right)$ & 4.0 & $148369.20^{*}$ & $\left.1\right|^{2} \mathrm{D}^{3} \mathrm{~F}$ \\
\hline 677.248 & -1.596 & 2.0 & $.00^{*}$ & $\left.\left.1\right|_{2} ^{3} \mathrm{~F}\right)$ & 3.0 & $147656.50^{*}$ & $\left.1\right|^{2} \mathrm{D}^{3} \mathrm{~F}$ \\
\hline 675.476 & -1.492 & 3.0 & $325.40^{*}$ & $\left.\left.1\right|_{2} ^{3} \mathrm{~F}\right)$ & 4.0 & $148369.20^{*}$ & $\left.1\right|^{2} \mathrm{D}^{3} \mathrm{~F}$ \\
\hline 661.192 & -2.842 & 3.0 & $325.40^{*}$ & $\left.\left.1\right|_{2} ^{3} \mathrm{~F}\right)$ & 2.0 & $151567.30^{*}$ & $\left.1\right|^{2} \mathrm{D}^{3} \mathrm{P}$ \\
\hline 660.384 & -3.106 & 2.0 & $.00 *$ & $\left.\left.1\right|_{2} ^{3} \mathrm{~F}\right)$ & 1.0 & $151427.00^{*}$ & $\left.1\right|^{2} \mathrm{D}^{3} \mathrm{P}$ \\
\hline 659.773 & -3.654 & 2.0 & $.00^{*}$ & $\left.\left.1\right|_{2} ^{3} \mathrm{~F}\right)$ & 2.0 & $151567.30^{*}$ & $\left.1\right|^{2} \mathrm{D}^{3} \mathrm{P}$ \\
\hline 652.810 & -2.944 & 4.0 & $734.70^{*}$ & $\left.\left.1\right|_{2} ^{3} \mathrm{~F}\right)$ & 3.0 & 153918.70* & $\left.1\right|^{2} \mathrm{D}^{1} \mathrm{~F}$ \\
\hline 651.070 & -3.342 & 3.0 & $325.40^{*}$ & $\left.\left.1\right|_{2} ^{3} \mathrm{~F}\right)$ & 3.0 & 153918.70* & $\left.1\right|^{2} \mathrm{D}^{1} \mathrm{~F}$ \\
\hline 649.694 & -3.943 & 2.0 & $.00^{*}$ & $\left.\left.1\right|_{2} ^{3} \mathrm{~F}\right)$ & 3.0 & $153918.70^{*}$ & $\left.1\right|^{2} \mathrm{D}^{1} \mathrm{~F}$ \\
\hline 642.816 & -3.339 & 2.0 & $.00 *$ & $\left.\left.1\right|_{2} ^{3} \mathrm{~F}\right)$ & 1.0 & $155565.50^{*}$ & $\left.1\right|^{2} \mathrm{D}^{1} \mathrm{P}$ \\
\hline
\end{tabular}

Table 5. Examples of differences for some $3 \mathrm{~d} 4 \mathrm{~s}-3 \mathrm{~d} 4 \mathrm{p}$ transitions in Ti III between log gf data obtained by our method and Kurucz data base compared to experimental intensity numbers by Edlén

\begin{tabular}{crrr}
$\lambda(\AA)$ & Kurucz & Edlén & Our work \\
\hline 2798.916 & -2.92 & 8 & -1.77 \\
2798.713 & -2.08 & 5 & -2.53 \\
2773.709 & -3.52 & 4 & -2.41 \\
2718.639 & -3.54 & 0 & -3.53 \\
2567.556 & -0.14 & 22 & -0.14 \\
2565.408 & -0.04 & 23 & 0.13 \\
2556.576 & -0.30 & 5 & -2.55 \\
2548.765 & -0.38 & 5 & -2.28 \\
2548.597 & -0.19 & 6 & -2.34
\end{tabular}


Table 6. Examples of differences for some $3 d^{2}-3 d 4 p$ transitions in Ti III between log gf data obtained by our method and Kurucz data base compared to experimental intensity numbers by Edlén

\begin{tabular}{|crrrr}
$\lambda(\AA)$ & Kurucz & Kelly & Edlén & Our work \\
\hline 1327.609 & -0.59 & 230 & 12 & -0.60 \\
1298.996 & -1.08 & 640 & 20 & -0.28 \\
1293.225 & -0.08 & 160 & 10 & -0.51 \\
1291.625 & -3.82 & 160 & 10 & -0.88 \\
1289.299 & -1.83 & 160 & 10 & -0.74 \\
1286.233 & -2.30 & 60 & 6 & -1.66 \\
1282.484 & -1.48 & 60 & 6 & -1.48
\end{tabular}

Table 7. Values for the electric quadrupole transition integrals in Ti III calculated by means of MCDF including core polarization

\begin{tabular}{c|cccccccc} 
& $3 \mathrm{~d}^{2}$ & $3 \mathrm{~d} 4 \mathrm{~s}$ & $4 \mathrm{~s}^{2}$ & $3 \mathrm{~d} 5 \mathrm{~s}$ & $3 \mathrm{~d} 6 \mathrm{~s}$ & $3 \mathrm{~d} 4 \mathrm{~d}$ & $3 \mathrm{~d} 5 \mathrm{~d}$ & $4 \mathrm{p}^{2}$ \\
\hline $3 \mathrm{~d}^{2}$ & 2.46 & -3.41 & - & .292 & .117 & -2.42 & -.990 & - \\
$3 \mathrm{~d} 4 \mathrm{~s}$ & -3.41 & 2.00 & -2.63 & - & - & 10.7 & 2.14 & - \\
$4 \mathrm{~s}^{2}$ & - & -2.63 & - & - & - & - & - & - \\
$3 \mathrm{~d} 5 \mathrm{~s}$ & .292 & - & - & 1.91 & - & -28.2 & 33.6 & - \\
$3 \mathrm{~d} 6 \mathrm{~s}$ & .117 & - & - & - & 1.89 & 8.19 & -89.8 & - \\
$3 \mathrm{~d} 4 \mathrm{~d}$ & -2.42 & 10.71 & - & -28.2 & 8.19 & 1.93 & -16.5 & - \\
& - & - & - & - & - & 26.2 & - & - \\
$3 \mathrm{~d} 5 \mathrm{~d}$ & -.990 & 2.14 & - & 33.6 & -89.8 & -16.5 & 1.91 & - \\
& - & - & - & - & - & - & 85.6 & - \\
$4 \mathrm{p}^{2}$ & - & - & - & - & - & - & - & 10.8
\end{tabular}

Table 8. Values for the electric quadrupole transition integrals in V IV calculated by means of MCDF including core polarization

\begin{tabular}{c|cccccccc} 
& $3 \mathrm{~d}^{2}$ & $3 \mathrm{~d} 4 \mathrm{~s}$ & $4 \mathrm{~s}^{2}$ & $3 \mathrm{~d} 5 \mathrm{~s}$ & $3 \mathrm{~d} 6 \mathrm{~s}$ & $3 \mathrm{~d} 4 \mathrm{~d}$ & $3 \mathrm{~d} 5 \mathrm{~d}$ & $4 \mathrm{p}^{2}$ \\
\hline $3 \mathrm{~d}^{2}$ & 1.64 & -2.12 & - & .040 & .015 & -1.53 & -.613 & - \\
$3 \mathrm{~d} 4 \mathrm{~s}$ & -2.12 & 1.41 & -1.73 & - & - & 8.06 & .775 & - \\
$4 \mathrm{~s}^{2}$ & - & -1.73 & - & - & - & - & - & - \\
$3 \mathrm{~d} 5 \mathrm{~s}$ & .040 & - & - & 1.37 & - & -16.4 & 25.3 & - \\
$3 \mathrm{~d} 6 \mathrm{~s}$ & .015 & - & - & - & 1.36 & 3.01 & -51.8 & - \\
$3 \mathrm{~d} 4 \mathrm{~d}$ & -1.53 & 8.06 & - & -16.4 & 3.01 & 1.38 & -9.66 & - \\
& - & - & - & - & - & 15.4 & - & - \\
$3 \mathrm{~d} 5 \mathrm{~d}$ & -.613 & .775 & - & 25.3 & -51.8 & -9.66 & 1.37 & - \\
& - & - & - & - & - & - & 49.9 & - \\
$4 \mathrm{p}^{2}$ & - & - & - & - & - & - & - & 7.80
\end{tabular}


Table 9. Calculated $A$-values for the $\left(3 \mathrm{~d}^{2}+3 \mathrm{~d} 4 \mathrm{~s}\right)-\left(3 \mathrm{~d}^{2}+3 \mathrm{~d} 4 \mathrm{~s}\right)$ M1 and E2 transition arrays of Ti III; the notation $x(y)$ means $x \times 10^{y}$

\begin{tabular}{|c|c|c|c|c|c|c|c|c|}
\hline$\lambda(\AA)$ & $A_{\mathrm{M} 1}\left(\mathrm{~s}^{-1}\right)$ & $A_{\mathrm{E} 2}\left(\mathrm{~s}^{-1}\right)$ & $J_{\mathrm{f}}$ & $E_{\mathrm{f}}\left(\mathrm{cm}^{-1}\right)$ & name & $J_{\mathrm{i}}$ & $E_{\mathrm{i}}\left(\mathrm{cm}^{-1}\right)$ & name \\
\hline 1533324.348 & $5.01(-6)$ & - & .0 & $10538.40^{*}$ & $\left.\left.1\right|_{2} ^{3} \mathrm{P}\right)$ & 1.0 & $10603.60^{*}$ & $\left.\left.1\right|_{2} ^{3} \mathrm{P}\right)$ \\
\hline 850108.397 & $2.19(-5)$ & $2.66(-12)$ & 1.0 & $10603.60^{*}$ & $\left.\left.1\right|_{2} ^{3} \mathrm{P}\right)$ & 2.0 & $10721.20^{*}$ & $\left.1 \mid{ }_{2}^{3} \mathrm{P}\right)$ \\
\hline 742739.580 & $5.93(-5)$ & $1.97(-12)$ & 1.0 & $38064.35 *$ & $\left.\left.2\right|_{1} ^{2} \mathrm{D}\right)^{3} \mathrm{D}$ & 2.0 & $38198.95^{*}$ & $\left.\left.2\right|_{1} ^{2} \mathrm{D}\right)^{3} \mathrm{D}$ \\
\hline 546896.868 & - & $1.07(-11)$ & .0 & $10538.40^{*}$ & $\left.\left.1\right|_{2} ^{3} \mathrm{P}\right)$ & 2.0 & $10721.20 *$ & $\left.\left.1\right|_{2} ^{3} \mathrm{P}\right)$ \\
\hline 540685.492 & $1.63(-4)$ & $1.61(-12)$ & 2.0 & $.00 *$ & $\left.\left.1\right|_{2} ^{3} \mathrm{~F}\right)$ & 3.0 & $184.90^{*}$ & $\left.\left.1\right|_{2} ^{3} \mathrm{~F}\right)$ \\
\hline 440330.988 & $2.11(-4)$ & $2.20(-11)$ & 2.0 & $38198.95^{*}$ & $\left.\left.2\right|_{1} ^{2} \mathrm{D}\right)^{3} \mathrm{D}$ & 3.0 & $38425.99^{*}$ & $\left.2 \mid{ }_{1}^{2} \mathrm{D}\right)^{3} \mathrm{D}$ \\
\hline 424512.728 & $2.65(-4)$ & $4.35(-12)$ & 3.0 & $184.90^{*}$ & $\left.\left.1\right|_{2} ^{3} \mathrm{~F}\right)$ & 4.0 & $420.40^{*}$ & $\left.\left.1\right|_{2} ^{3} \mathrm{~F}\right)$ \\
\hline 276442.726 & - & $2.26(-11)$ & 1.0 & $38064.35^{*}$ & $\left.\left.2\right|_{1} ^{2} \mathrm{D}\right)^{3} \mathrm{D}$ & 3.0 & $38425.99^{*}$ & $\left.2 \mid{ }_{1}^{2} \mathrm{D}\right)^{3} \mathrm{D}$ \\
\hline 237803.871 & - & $3.53(-12)$ & 2.0 & $.00 *$ & $\left.\left.1\right|_{2} ^{3} \mathrm{~F}\right)$ & 4.0 & $420.40 *$ & $\left.\left.1\right|_{2} ^{3} \mathrm{~F}\right)$ \\
\hline 48415.294 & - & $5.20(-8)$ & .0 & $10538.40^{*}$ & $\left.1 \mid{ }_{2}^{3} \mathrm{P}\right)$ & 2.0 & $8473.50 *$ & $1\left({ }_{2}^{1} \mathrm{D}\right)$ \\
\hline 46933.356 & $1.31(-3)$ & $7.61(-8)$ & 1.0 & $10603.60^{*}$ & $\left.\left.1\right|_{2} ^{3} \mathrm{P}\right)$ & 2.0 & $8473.50 *$ & $\left.\left.1\right|_{2} ^{1} \mathrm{D}\right)$ \\
\hline 44477.795 & $2.76(-3)$ & $1.32(-10)$ & 2.0 & $8473.50 *$ & $\left.1 \mid{ }_{2}^{\mathrm{T}} \mathrm{D}\right)$ & 2.0 & $10721.20 *$ & $\left.1{ }_{2}^{3} \mathrm{P}\right)$ \\
\hline 30495.483 & $2.41(-3)$ & $5.13(-8)$ & 2.0 & $41704.27^{*}$ & $\left.2 \mid{ }_{1}^{2} \mathrm{D}\right){ }^{1} \mathrm{D}$ & 3.0 & $38425.99^{*}$ & $\left.\left.2\right|_{1} ^{2} \mathrm{D}\right)^{3} \mathrm{D}$ \\
\hline 28520.286 & $5.25(-4)$ & $8.70(-8)$ & 2.0 & $38198.95^{*}$ & $\left.\left.2\right|_{1} ^{2} \mathrm{D}\right)^{3} \mathrm{D}$ & 2.0 & $41704.27^{*}$ & $\left.2 \mid{ }_{1}^{2} \mathrm{D}\right){ }^{1} \mathrm{D}$ \\
\hline 27465.639 & $3.18(-3)$ & $7.63(-8)$ & 1.0 & $38064.35 *$ & $\left.\left.2\right|_{1} ^{\frac{1}{2}} \mathrm{D}\right)^{3} \mathrm{D}$ & 2.0 & $41704.27^{*}$ & $\left.\left.2\right|_{1} ^{\frac{1}{2}} \mathrm{D}\right){ }^{1} \mathrm{D}$ \\
\hline 27193.104 & - & $2.52(-7)$ & 2.0 & $10721.20^{*}$ & $\left.\left.1\right|_{2} ^{3} \mathrm{P}\right)$ & 4.0 & $14397.60^{*}$ & $\left.1 \mid{ }_{2}^{\dagger} \mathrm{G}\right)$ \\
\hline 17887.884 & $5.18(-16)$ & - & .0 & $32475.50^{*}$ & $\left.1{ }_{0}^{1} \mathrm{~S}\right)$ & 1.0 & $38064.35^{*}$ & $\left.2 \mid{ }_{1}^{2} \mathrm{D}\right)^{3} \mathrm{D}$ \\
\hline 17467.209 & - & $8.65(-7)$ & .0 & $32475.50 *$ & $\left.\left.1\right|_{0} ^{\mathrm{Y}} \mathrm{S}\right)$ & 2.0 & $38198.95^{*}$ & $\left.2 \mid{ }_{1}^{2} \mathrm{D}\right)^{3} \mathrm{D}$ \\
\hline 16875.592 & - & $3.22(-4)$ & 2.0 & $8473.50 *$ & $\left.1 \mid{ }_{2}^{Y} \mathrm{D}\right)$ & 4.0 & $14397.60^{*}$ & $1\left({ }_{2}^{\dagger} \mathrm{G}\right)$ \\
\hline 12414.182 & - & $5.66(-5)$ & 2.0 & $8473.50 *$ & $\left.\left.1\right|_{2} ^{1} \mathrm{D}\right)$ & 4.0 & $420.40 *$ & $\left.\left.1\right|_{2} ^{3} \mathrm{~F}\right)$ \\
\hline 12061.463 & $1.02(-2)$ & $1.17(-5)$ & 2.0 & $8473.50 *$ & $\left.\left.1\right|_{2} ^{\mathbb{T}} \mathrm{D}\right)$ & 3.0 & $184.90 *$ & $\left.\left.1\right|_{2} ^{3} \mathrm{~F}\right)$ \\
\hline 11798.270 & $5.46(-3)$ & $1.18(-5)$ & 2.0 & $.00^{*}$ & $\left.\left.1\right|_{2} ^{3} \mathrm{~F}\right)$ & 2.0 & $8473.50 *$ & $\left.\left.1\right|_{2} ^{1} \mathrm{D}\right)$ \\
\hline 10832.713 & - & $6.18(-3)$ & .0 & $32475.50 *$ & $\left.\left.1\right|_{0} ^{4} \mathrm{~S}\right)$ & 2.0 & $41704.27^{*}$ & $\left.\left.2\right|_{1} ^{2} \mathrm{D}\right){ }^{1} \mathrm{D}$ \\
\hline 9705.322 & - & $2.77(-2)$ & 2.0 & $10721.20^{*}$ & $\left.\left.1\right|_{2} ^{3} \mathrm{P}\right)$ & 4.0 & $420.40 *$ & $\left.\left.1\right|_{2} ^{3} \mathrm{~F}\right)$ \\
\hline 9595.495 & - & $2.55(-2)$ & 1.0 & $10603.60^{*}$ & $\left.\left.1\right|_{2} ^{3} \mathrm{P}\right)$ & 3.0 & $184.90^{*}$ & $\left.\left.1\right|_{2} ^{3} \mathrm{~F}\right)$ \\
\hline 9488.395 & $7.99(-5)$ & $8.05(-3)$ & 2.0 & $10721.20^{*}$ & $\left.\left.1\right|_{2} ^{3} \mathrm{P}\right)$ & 3.0 & $184.90^{*}$ & $\left.1 \mid{ }_{2}^{3} \mathrm{~F}\right)$ \\
\hline 9486.504 & - & $4.05(-2)$ & .0 & $10538.40^{*}$ & $\left.\left.1\right|_{2} ^{3} \mathrm{P}\right)$ & 2.0 & $.00^{*}$ & $\left.\left.1\right|_{2} ^{3} \mathrm{~F}\right)$ \\
\hline 9428.173 & $3.41(-6)$ & $1.39(-2)$ & 1.0 & $10603.60^{*}$ & $\left.\left.1\right|_{2} ^{3} \mathrm{P}\right)$ & 2.0 & $.00 *$ & $\left.\left.1\right|_{2} ^{3} \mathrm{~F}\right)$ \\
\hline 9324.756 & $1.58(-5)$ & $1.24(-3)$ & 2.0 & $.00 *$ & $\left.\left.1\right|_{2} ^{3} \mathrm{~F}\right)$ & 2.0 & $10721.20 *$ & $\left.\left.1\right|_{2} ^{3} \mathrm{P}\right)$ \\
\hline 7152.537 & $7.13(-3)$ & $9.32(-6)$ & 4.0 & $420.40^{*}$ & $\left.\left.1\right|_{2} ^{3} \mathrm{~F}\right)$ & 4.0 & $14397.60^{*}$ & $\left.\left.1\right|_{2} ^{1} G\right)$ \\
\hline 7034.021 & $4.50(-3)$ & $2.65(-7)$ & 3.0 & $184.90^{*}$ & $\left.\left.1\right|_{2} ^{3} \mathrm{~F}\right)$ & 4.0 & $14397.60^{*}$ & $\left.\left.1\right|_{2} ^{1} \mathrm{G}\right)$ \\
\hline 6943.687 & - & $1.56(-5)$ & 2.0 & $.00 *$ & $\left.\left.1\right|_{2} ^{3} \mathrm{~F}\right)$ & 4.0 & $14397.60^{*}$ & $\left.\left.1\right|_{2} ^{1} \mathrm{G}\right)$ \\
\hline 4595.505 & - & $2.23(-2)$ & .0 & $32475.50 *$ & $\left.\left.1\right|_{0} ^{1} \mathrm{~S}\right)$ & 2.0 & $10721.20 *$ & $\left.\left.1\right|_{2} ^{3} \mathrm{P}\right)$ \\
\hline 4570.795 & $8.87(-2)$ & - & .0 & $32475.50 *$ & $\left.\left.1\right|_{0} ^{\mathrm{I}} \mathrm{S}\right)$ & 1.0 & $10603.60^{*}$ & $\left.1 \mid{ }_{2}^{3} \mathrm{P}\right)$ \\
\hline 4200.259 & - & $1.76(-2)$ & 2.0 & $38198.95^{*}$ & $\left.2 \mid{ }_{1}^{2} \mathrm{D}\right)^{3} \mathrm{D}$ & 4.0 & $14397.60^{*}$ & $\left.\left.1\right|_{2} ^{1} \mathrm{G}\right)$ \\
\hline 4165.145 & - & $4.95(0)$ & .0 & $32475.50 *$ & $\left.\left.1\right|_{0} ^{\mathrm{t}} \mathrm{S}\right)$ & 2.0 & $8473.50^{*}$ & $\left.\left.1\right|_{2} ^{4} \mathrm{D}\right)$ \\
\hline 4160.571 & $2.48(-14)$ & $3.39(-4)$ & 3.0 & $38425.99^{*}$ & $\left.\left.2\right|_{1} ^{2} \mathrm{D}\right)^{3} \mathrm{D}$ & 4.0 & $14397.60 *$ & $\left.\left.1\right|_{2} ^{1} \mathrm{G}\right)$ \\
\hline 3661.066 & - & $1.48(1)$ & 2.0 & $41704.27^{*}$ & $\left.\left.2\right|_{1} ^{2} \mathrm{D}\right)^{1} \mathrm{D}$ & 4.0 & $14397.60^{*}$ & $\left.\left.1\right|_{2} ^{\mathrm{T}} \mathrm{G}\right)$ \\
\hline 3656.181 & $6.25(-7)$ & $1.19(0)$ & 1.0 & $38064.35^{*}$ & $\left.2 \mid{ }_{1}^{\frac{1}{2}} \mathrm{D}\right)^{3} \mathrm{D}$ & 2.0 & $10721.20^{*}$ & $\left.\left.1\right|_{2} ^{3} \mathrm{P}\right)$ \\
\hline 3640.524 & $6.41(-10)$ & $3.69(0)$ & 1.0 & $10603.60 *$ & $\left.\left.1\right|_{2} ^{3} \mathrm{P}\right)$ & 1.0 & $38064.35^{*}$ & $\left.\left.2\right|_{1} ^{2} \mathrm{D}\right)^{3} \mathrm{D}$ \\
\hline 3638.271 & $6.21(-8)$ & $2.82(0)$ & 2.0 & $10721.20^{*}$ & $\left.\left.1\right|_{2} ^{3} \mathrm{P}\right)$ & 2.0 & $38198.95^{*}$ & $\left.\left.2\right|_{1} ^{2} \mathrm{D}\right)^{3} \mathrm{D}$ \\
\hline 3631.900 & $2.15(-10)$ & - & .0 & $10538.40^{*}$ & $\left.\left.1\right|_{2} ^{3} \mathrm{P}\right)$ & 1.0 & $38064.35^{*}$ & $\left.2 \mid{ }_{1}^{2} \mathrm{D}\right)^{3} \mathrm{D}$ \\
\hline 3622.766 & $9.85(-8)$ & $4.19(-1)$ & 1.0 & $10603.60^{*}$ & $\left.1 \mid{ }_{2}^{3} \mathrm{P}\right)$ & 2.0 & $38198.95^{*}$ & $\left.\left.2\right|_{1} ^{2} \mathrm{D}\right)^{3} \mathrm{D}$ \\
\hline 3614.226 & - & $1.70(0)$ & .0 & $10538.40^{*}$ & $\left.\left.1\right|_{2} ^{3} \mathrm{P}\right)$ & 2.0 & $38198.95^{*}$ & $\left.\left.2\right|_{1} ^{2} \mathrm{D}\right){ }^{3} \mathrm{D}$ \\
\hline 3608.455 & $2.77(-7)$ & $3.40(0)$ & 2.0 & $10721.20^{*}$ & $\left.\left.1\right|_{2} ^{3} \mathrm{P}\right)$ & 3.0 & $38425.99^{*}$ & $\left.\left.2\right|_{1} ^{2} \mathrm{D}\right)^{3} \mathrm{D}$ \\
\hline 3593.202 & - & $1.75(0)$ & 1.0 & $10603.60^{*}$ & $\left.\left.1\right|_{2} ^{3} \mathrm{P}\right)$ & 3.0 & $38425.99^{*}$ & $\left.\left.2\right|_{1} ^{2} \mathrm{D}\right)^{3} \mathrm{D}$ \\
\hline 3378.453 & $1.00(-4)$ & $3.12(-2)$ & 1.0 & $38064.35^{*}$ & $\left.2 \mid{ }_{1}^{2} \mathrm{D}\right)^{3} \mathrm{D}$ & 2.0 & $8473.50^{*}$ & $\left.\left.1\right|_{2} ^{1} \mathrm{D}\right)$ \\
\hline 3363.154 & $1.16(-5)$ & $799(-2)$ & 2.0 & $8473.50^{*}$ & $\left.\left.1\right|_{2} ^{1} \mathrm{D}\right)$ & 2.0 & $38198.95^{*}$ & $\left.\left.2\right|_{1} ^{2} \mathrm{D}\right)^{3} \mathrm{D}$ \\
\hline 3337.661 & $4.63(-5)$ & $3.54(-2)$ & 2.0 & $8473.50^{*}$ & $\left.\left.1\right|_{2} ^{4} \mathrm{D}\right)$ & 3.0 & $38425.99^{*}$ & $\left.\left.2\right|_{1} ^{2} \mathrm{D}\right)^{3} \mathrm{D}$ \\
\hline 3226.638 & $9.67(-5)$ & $1.62(-1)$ & 2.0 & $10721.20^{*}$ & $\left.1 \mid{ }_{2}^{3} \mathrm{P}\right)$ & 2.0 & $41704.27^{*}$ & $\left.2 \mid{ }_{1}^{2} \mathrm{D}\right){ }^{1} \mathrm{D}$ \\
\hline 3214.436 & $3.27(-5)$ & $2.09(-3)$ & 1.0 & $10603.60^{*}$ & $\left.1 \mid{ }_{2}^{3} \mathrm{P}\right)$ & 2.0 & $41704.27^{*}$ & $\left.\left.2\right|_{1} ^{2} \mathrm{D}\right){ }^{1} \mathrm{D}$ \\
\hline 3207.711 & - & $5.00(-3)$ & .0 & $10538.40^{*}$ & $\left.\left.1\right|_{2} ^{3} \mathrm{P}\right)$ & 2.0 & $41704.27^{*}$ & $\left.\left.2\right|_{1} ^{2} \mathrm{D}\right)^{1} \mathrm{D}$ \\
\hline 3078.350 & - & $4.17(-3)$ & .0 & $32475.50^{*}$ & $\left.1{ }_{0}^{\mathrm{T}} \mathrm{S}\right)$ & 2.0 & $.00 *$ & $\left.\left.1\right|_{2} ^{3} \mathrm{~F}\right)$ \\
\hline 3008.382 & $5.97(-7)$ & $1.89(1)$ & 2.0 & $8473.50^{*}$ & & 2.0 & $41704.27^{*}$ & $\left.\left.2\right|_{1} ^{2} \mathrm{D}\right)^{1} \mathrm{D}$ \\
\hline 2646.217 & - & $1.18(1)$ & 2.0 & $38198.95^{*}$ & $\left.2 \mid{ }_{1}^{2} \mathrm{D}\right)^{3} \mathrm{D}$ & 4.0 & $420.40^{*}$ & $\left.\left.1\right|_{2} ^{3} \mathrm{~F}\right)$ \\
\hline 2639.167 & - & $1.86(1)$ & 1.0 & $38064.35^{*}$ & $\left.\left.2\right|_{1} ^{2} \mathrm{D}\right)^{3} \mathrm{D}$ & 3.0 & $184.90^{*}$ & $\left.\left.1\right|_{2} ^{3} \mathrm{~F}\right)$ \\
\hline 2630.408 & $1.52(-9)$ & $4.35(1)$ & 3.0 & $38425.99^{*}$ & $\left.2 \mid{ }_{1}^{2} \mathrm{D}\right)^{3} \mathrm{D}$ & 4.0 & $420.40^{*}$ & $\left.1{ }_{2}^{3} \mathrm{~F}\right)$ \\
\hline 2629.822 & $4.14(-7)$ & $2.84(1)$ & 2.0 & $38198.95 *$ & $\left.\left.2\right|_{1} ^{2} \mathrm{D}\right)^{3} \mathrm{D}$ & 3.0 & $184.90^{*}$ & $\left.\left.1\right|_{2} ^{3} \mathrm{~F}\right)$ \\
\hline 2626.347 & $5.95(-8)$ & $3.82(1)$ & 1.0 & $38064.35^{*}$ & $\left.2 \mid{ }_{1}^{2} \mathrm{D}\right)^{3} \mathrm{D}$ & 2.0 & $.00^{*}$ & $\left.1 \mid{ }_{2}^{3} \mathrm{~F}\right)$ \\
\hline 2617.092 & $1.22(-7)$ & $1.67(1)$ & 2.0 & $.00 *$ & $\left.\left.1\right|_{2} ^{3} \mathrm{~F}\right)$ & 2.0 & $38198.95^{*}$ & $\left.2 \mid{ }_{1}^{2} \mathrm{D}\right)^{3} \mathrm{D}$ \\
\hline 2614.208 & $1.20(-9)$ & $1.26(1)$ & 3.0 & $184.90 *$ & $\left.\left.1\right|_{2} ^{3} \mathrm{~F}\right)$ & 3.0 & $38425.99^{*}$ & $\left.\left.2\right|_{1} ^{2} \mathrm{D}\right)^{3} \mathrm{D}$ \\
\hline 2601.628 & $1.58(-8)$ & $1.22(0)$ & 2.0 & $.00 *$ & $\left.\left.1\right|_{2} ^{3} \mathrm{~F}\right)$ & 3.0 & $38425.99^{*}$ & $\left.\left.2\right|_{1} ^{2} \mathrm{D}\right)^{3} \mathrm{D}$ \\
\hline 2421.518 & - & $1.68(-2)$ & 2.0 & $41704.27^{*}$ & $\left.2 \mid{ }_{1}^{2} \mathrm{D}\right)^{1} \mathrm{D}$ & 4.0 & $420.40^{*}$ & $\left.\left.1\right|_{2} ^{3} \mathrm{~F}\right)$ \\
\hline 2407.782 & $1.33(-4)$ & $1.19(-1)$ & 2.0 & $41704.27^{*}$ & $\left.2 \mid{ }_{1}^{2} \mathrm{D}\right)^{1} \mathrm{D}$ & 3.0 & $184.90^{*}$ & $\left.1{ }_{2}^{3} \mathrm{~F}\right)$ \\
\hline 2397.106 & $6.75(-5)$ & $8.33(-3)$ & 2.0 & $.00 *$ & $\left.\left.1\right|_{{ }_{2}^{3}} ^{1} \mathrm{~F}\right)$ & 2.0 & $41704.27^{*}$ & $\left.2{ }_{1}^{2} \mathrm{D}\right)^{1} \mathrm{D}$ \\
\hline
\end{tabular}


Table 10. Calculated $A$-values for the $\left(3 \mathrm{~d}^{2}+3 \mathrm{~d} 4 \mathrm{~s}\right)-\left(3 \mathrm{~d}^{2}+3 \mathrm{~d} 4 \mathrm{~s}\right)$ M1 and E2 transition arrays of V IV; the notation $x(y)$ means $x \times 10^{y}$

\begin{tabular}{|c|c|c|c|c|c|c|c|c|}
\hline$\lambda(\AA)$ & $A_{\mathrm{M} 1}\left(\mathrm{~s}^{-1}\right)$ & $A_{\mathrm{E} 2}\left(\mathrm{~s}^{-1}\right)$ & $J_{\mathrm{f}}$ & $E_{\mathrm{f}}\left(\mathrm{cm}^{-1}\right)$ & name & $J_{\mathrm{i}}$ & $E_{\mathrm{i}}\left(\mathrm{cm}^{-1}\right)$ & name \\
\hline 858872.401 & $2.85(-5)$ & - & .0 & $13122.80^{*}$ & $\left.\left.1\right|_{2} ^{3} \mathrm{P}\right)$ & 1.0 & $13239.20^{*}$ & $\left.\left.1\right|_{2} ^{3} \mathrm{P}\right)$ \\
\hline 462836.794 & $2.44(-4)$ & $1.04(-11)$ & 1.0 & $96196.10^{*}$ & $\left.\left.2\right|_{1} ^{2} \mathrm{D}\right)^{3} \mathrm{D}$ & 2.0 & $96412.10^{*}$ & $\left.\left.2\right|_{1} ^{2} \mathrm{D}\right)^{3} \mathrm{D}$ \\
\hline 456288.213 & $1.40(-4)$ & $2.72(-11)$ & 1.0 & $13239.20^{*}$ & $\left.\left.1\right|_{2} ^{3} \mathrm{P}\right)$ & 2.0 & $13458.30^{*}$ & $\left.\left.1\right|_{2} ^{3} \mathrm{P}\right)$ \\
\hline 307230.324 & $8.88(-4)$ & $1.13(-11)$ & 2.0 & $.00 *$ & $\left.\left.1\right|_{2} ^{3} \mathrm{~F}\right)$ & 3.0 & $325.40^{*}$ & $\left.\left.1\right|_{2} ^{3} \mathrm{~F}\right)$ \\
\hline 297981.363 & - & $1.02(-10)$ & .0 & $13122.80^{*}$ & $\left.\left.1\right|_{2} ^{3} \mathrm{P}\right)$ & 2.0 & $13458.30^{*}$ & $\left.\left.1\right|_{2} ^{3} \mathrm{P}\right)$ \\
\hline 259063.870 & $1.03(-3)$ & $1.54(-10)$ & 2.0 & $96412.10^{*}$ & $\left.\left.2\right|_{1} ^{2} \mathrm{D}\right)^{3} \mathrm{D}$ & 3.0 & $96798.00^{*}$ & $\left.\left.2\right|_{1} ^{2} \mathrm{D}\right)^{3} \mathrm{D}$ \\
\hline 244252.986 & $1.39(-3)$ & $2.88(-11)$ & 3.0 & $325.40^{*}$ & $\left.\left.1\right|_{2} ^{3} \mathrm{~F}\right)$ & 4.0 & $734.70^{*}$ & $\left.\left.1\right|_{2} ^{3} \mathrm{~F}\right)$ \\
\hline 166095.277 & - & $1.43(-10)$ & 1.0 & 96196.10* & $\left.2 \mid{ }_{1}^{2} \mathrm{D}\right)^{3} \mathrm{D}$ & 3.0 & 96798.00* & $\left.\left.2\right|_{1} ^{2} \mathrm{D}\right)^{3} \mathrm{D}$ \\
\hline 136072.882 & - & $2.35(-11)$ & 2.0 & $.00 *$ & $\left.\left.1\right|_{2} ^{3} \mathrm{~F}\right)$ & 4.0 & $734.70^{*}$ & $\left.\left.1\right|_{2} ^{3} \mathrm{~F}\right)$ \\
\hline 46208.801 & - & $8.40(-8)$ & .0 & $13122.80^{*}$ & $\left.\left.1\right|_{2} ^{3} \mathrm{P}\right)$ & 2.0 & 10959.30* & $\left.\left.1\right|_{2} ^{\mathrm{T}} \mathrm{D}\right)$ \\
\hline 43849.616 & $4.09(-3)$ & $1.18(-7)$ & 1.0 & $13239.20^{*}$ & $\left.\left.1\right|_{2} ^{3} \mathrm{P}\right)$ & 2.0 & 10959.30* & $\left.\left.1\right|_{2} ^{1} \mathrm{D}\right)$ \\
\hline 40005.097 & $9.55(-3)$ & $5.34(-9)$ & 2.0 & 10959.30* & $\left.\left.1\right|_{2} ^{\mathrm{T}} \mathrm{D}\right)$ & 2.0 & $13458.30 *$ & $\left.\left.1\right|_{2} ^{3} \mathrm{P}\right)$ \\
\hline 29380.413 & $6.32(-3)$ & $7.34(-8)$ & 2.0 & $100200.70^{*}$ & $\left.\left.2\right|_{1} ^{2} \mathrm{D}\right){ }^{1} \mathrm{D}$ & 3.0 & $96798.00^{*}$ & $\left.\left.2\right|_{1} ^{2} \mathrm{D}\right)^{3} \mathrm{D}$ \\
\hline 26387.775 & $1.55(-3)$ & $8.30(-8)$ & 2.0 & $96412.10^{*}$ & $\left.2 \mid{ }_{1}^{2} \mathrm{D}\right)^{3} \mathrm{D}$ & 2.0 & $100200.70^{*}$ & $\left.\left.2\right|_{1} ^{2} \mathrm{D}\right)^{1} \mathrm{D}$ \\
\hline 24964.472 & $9.93(-3)$ & $1.45(-7)$ & 1.0 & $96196.10^{*}$ & $\left.\left.2\right|_{1} ^{2} \mathrm{D}\right)^{3} \mathrm{D}$ & 2.0 & $100200.70^{*}$ & $\left.2 \mid{ }_{1}^{2} \mathrm{D}\right)^{1} \mathrm{D}$ \\
\hline 20266.519 & - & $1.61(-6)$ & 2.0 & $13458.30 *$ & $\left.\left.1\right|_{2} ^{3} \mathrm{P}\right)$ & 4.0 & $18391.20^{*}$ & $\left.1 \mid{ }_{2}^{\dagger} \mathrm{G}\right)$ \\
\hline 13451.831 & - & $6.17(-4)$ & 2.0 & $10959.30^{*}$ & $\left.\left.1\right|_{2} ^{\mathrm{T}} \mathrm{D}\right)$ & 4.0 & $18391.20 *$ & $\left.\left.1\right|_{2} ^{\mathrm{T}} \mathrm{G}\right)$ \\
\hline 9777.652 & - & $2.09(-4)$ & 2.0 & 10959.30* & $\left.\left.1\right|_{2} ^{\mathrm{T}} \mathrm{D}\right)$ & 4.0 & $734.70^{*}$ & $\left.\left.1\right|_{2} ^{3} \mathrm{~F}\right)$ \\
\hline 9401.308 & $3.92(-2)$ & $4.64(-5)$ & 2.0 & $10959.30^{*}$ & $\left.\left.1\right|_{2} ^{\mathrm{T}} \mathrm{D}\right)$ & 3.0 & $325.40^{*}$ & $\left.\left.1\right|_{2} ^{3} \mathrm{~F}\right)$ \\
\hline 9122.167 & $2.16(-2)$ & $3.39(-5)$ & 2.0 & $.00 *$ & $\left.\left.1\right|_{2} ^{3} \mathrm{~F}\right)$ & 2.0 & $10959.30 *$ & $\left.1 \mid{ }_{2}^{1} \mathrm{D}\right)$ \\
\hline 7857.249 & - & $3.46(-2)$ & 2.0 & $13458.30 *$ & $\left.\left.1\right|_{2} ^{3} \mathrm{P}\right)$ & 4.0 & $734.70^{*}$ & $\left.\left.1\right|_{2} ^{3} \mathrm{~F}\right)$ \\
\hline 7741.524 & - & $3.28(-2)$ & 1.0 & $13239.20^{*}$ & $\left.\left.1\right|_{2} ^{3} \mathrm{P}\right)$ & 3.0 & $325.40^{*}$ & $\left.\left.1\right|_{2} ^{3} \mathrm{~F}\right)$ \\
\hline 7618.228 & - & $5.34(-2)$ & .0 & $13122.80^{*}$ & $\left.\left.1\right|_{2} ^{3} \mathrm{P}\right)$ & 2.0 & $.00 *$ & $\left.\left.1\right|_{2} ^{3} \mathrm{~F}\right)$ \\
\hline 7612.369 & $7.64(-4)$ & $1.06(-2)$ & 2.0 & $13458.30^{*}$ & $\left.\left.1\right|_{2} ^{3} \mathrm{P}\right)$ & 3.0 & $325.40^{*}$ & $\left.\left.1\right|_{2} ^{3} \mathrm{~F}\right)$ \\
\hline 7551.247 & $2.41(-5)$ & $1.85(-2)$ & 1.0 & $13239.20^{*}$ & $\left.\left.1\right|_{2} ^{3} \mathrm{P}\right)$ & 2.0 & $.00 *$ & $\left.\left.1\right|_{2} ^{3} \mathrm{~F}\right)$ \\
\hline 7428.313 & $1.84(-4)$ & $1.66(-3)$ & 2.0 & $.00^{*}$ & $\left.\left.1\right|_{2} ^{3} \mathrm{~F}\right)$ & 2.0 & $13458.30^{*}$ & $\left.\left.1\right|_{2} ^{3} \mathrm{P}\right)$ \\
\hline 5662.065 & $2.76(-2)$ & $2.88(-5)$ & 4.0 & $734.70^{*}$ & $\left.\left.1\right|_{2} ^{3} \mathrm{~F}\right)$ & 4.0 & $18391.20 *$ & $\left.\left.1\right|_{2} ^{\mathrm{T}} \mathrm{G}\right)$ \\
\hline 5533.784 & $1.78(-2)$ & $7.14(-7)$ & 3.0 & $325.40 *$ & $\left.\left.1\right|_{2} ^{3} \mathrm{~F}\right)$ & 4.0 & $18391.20^{*}$ & $\left.\left.1\right|_{2} ^{T} \mathrm{G}\right)$ \\
\hline 5435.872 & - & $5.61(-5)$ & 2.0 & $.00 *$ & $\left.\left.1\right|_{2} ^{3} \mathrm{~F}\right)$ & 4.0 & $18391.20^{*}$ & $\left.\left.1\right|_{2} ^{T} \mathrm{G}\right)$ \\
\hline 3446.836 & - & $1.32(-1)$ & .0 & $42462.10^{*}$ & $\left.\left.1\right|_{0} ^{1} \mathrm{~S}\right)$ & 2.0 & $13458.30^{*}$ & $\left.\left.1\right|_{2} ^{3} \mathrm{P}\right)$ \\
\hline 3420.993 & $3.87(-1)$ & - & .0 & $42462.10^{*}$ & $\left.\left.1\right|_{0} ^{\mathrm{T}} \mathrm{S}\right)$ & 1.0 & $13239.20^{*}$ & $\left.\left.1\right|_{2} ^{3} \mathrm{P}\right)$ \\
\hline 3173.403 & - & $1.11(1)$ & .0 & $42462.10^{*}$ & $\left.\left.1\right|_{0} ^{1} \mathrm{~S}\right)$ & 2.0 & 10959.30* & $\left.\left.1\right|_{2} ^{\mathrm{I}} \mathrm{D}\right)$ \\
\hline 2354.321 & - & $1.96(-2)$ & .0 & $42462.10 *$ & $\left.\left.1\right|_{0} ^{1} \mathrm{~S}\right)$ & 2.0 & $.00 *$ & $\left.\left.1\right|_{2} ^{3} \mathrm{~F}\right)$ \\
\hline 1861.019 & $4.59(-14)$ & - & .0 & $42462.10^{*}$ & $\left.\left.1\right|_{0} ^{1} \mathrm{~S}\right)$ & 1.0 & $96196.10^{*}$ & $\left.2 \mid{ }_{1}^{2} \mathrm{D}\right)^{3} \mathrm{D}$ \\
\hline 1853.568 & - & $5.84(-2)$ & .0 & $42462.10^{*}$ & $\left.\left.1\right|_{0} ^{1} \mathrm{~S}\right)$ & 2.0 & $96412.10^{*}$ & $\left.2 \mid{ }_{1}^{2} \mathrm{D}\right)^{3} \mathrm{D}$ \\
\hline 1731.944 & - & $2.16(1)$ & .0 & $42462.10^{*}$ & $\left.\left.1\right|_{0} ^{1} \mathrm{~S}\right)$ & 2.0 & $100200.70^{*}$ & $\left.2 \mid{ }_{1}^{2} \mathrm{D}\right)^{1} \mathrm{D}$ \\
\hline 1281.708 & - & $5.73(0)$ & 2.0 & $96412.10 *$ & $\left.\left.2\right|_{1} ^{2} \mathrm{D}\right)^{3} \mathrm{D}$ & 4.0 & $18391.20^{*}$ & $\left.\left.1\right|_{2} ^{\dagger} \mathrm{G}\right)$ \\
\hline 1275.400 & $1.15(-13)$ & $9.60(-2)$ & 3.0 & 96798.00* & $\left.\left.2\right|_{1} ^{2} \mathrm{D}\right)^{3} \mathrm{D}$ & 4.0 & $18391.20^{*}$ & $\left.\left.1\right|_{2} ^{T} G\right)$ \\
\hline 1222.352 & - & $1.30(3)$ & 2.0 & $100200.70^{*}$ & $\left.2 \mid{ }_{1}^{2} \mathrm{D}\right){ }^{1} \mathrm{D}$ & 4.0 & $18391.20^{*}$ & $\left.\left.1\right|_{2} ^{1} \mathrm{G}\right)$ \\
\hline 1208.637 & $1.43(-7)$ & $1.11(2)$ & 1.0 & $96196.10^{*}$ & $\left.\left.2\right|_{1} ^{2} \mathrm{D}\right)^{3} \mathrm{D}$ & 2.0 & $13458.30^{*}$ & $\left.\left.1\right|_{2} ^{3} \mathrm{P}\right)$ \\
\hline 1205.490 & $1.19(-8)$ & $2.59(2)$ & 2.0 & $13458.30 *$ & $\left.\left.1\right|_{2} ^{3} \mathrm{P}\right)$ & 2.0 & $96412.10^{*}$ & $\left.\left.2\right|_{1} ^{2} \mathrm{D}\right)^{3} \mathrm{D}$ \\
\hline 1205.445 & $7.30(-10)$ & $3.49(2)$ & 1.0 & $13239.20^{*}$ & $\left.\left.1\right|_{2} ^{3} \mathrm{P}\right)$ & 1.0 & $96196.10^{*}$ & $\left.2 \mid{ }_{1}^{2} \mathrm{D}\right)^{3} \mathrm{D}$ \\
\hline 1203.756 & $2.44(-10)$ & - & .0 & $13122.80^{*}$ & $\left.\left.1\right|_{2} ^{3} \mathrm{P}\right)$ & 1.0 & $96196.10^{*}$ & $\left.\left.2\right|_{1} ^{2} \mathrm{D}\right)^{3} \mathrm{D}$ \\
\hline 1202.315 & $2.33(-8)$ & $3.91(1)$ & 1.0 & $13239.20^{*}$ & $\left.\left.1\right|_{2} ^{3} \mathrm{P}\right)$ & 2.0 & $96412.10^{*}$ & $\left.\left.2\right|_{1} ^{\frac{1}{2}} \mathrm{D}\right)^{3} \mathrm{D}$ \\
\hline 1200.634 & - & $1.58(2)$ & .0 & $13122.80 *$ & $\left.\left.1\right|_{2} ^{3} \mathrm{P}\right)$ & 2.0 & $96412.10^{*}$ & $\left.\left.2\right|_{1} ^{2} \mathrm{D}\right)^{3} \mathrm{D}$ \\
\hline 1199.908 & $5.94(-8)$ & $3.12(2)$ & 2.0 & $13458.30^{*}$ & $\left.\left.1\right|_{2} ^{3} \mathrm{P}\right)$ & 3.0 & $96798.00^{*}$ & $\left.\left.2\right|_{1} ^{\frac{1}{2}} \mathrm{D}\right)^{3} \mathrm{D}$ \\
\hline 1196.762 & - & $1.61(2)$ & 1.0 & $13239.20^{*}$ & $\left.\left.1\right|_{2} ^{3} \mathrm{P}\right)$ & 3.0 & 96798.00* & $\left.\left.2\right|_{1} ^{2} \mathrm{D}\right)^{3} \mathrm{D}$ \\
\hline 1173.202 & $7(-6)$ & $532(0)$ & 1.0 & $96196.10^{*}$ & $\left.\left.2\right|_{1} ^{2} \mathrm{D}\right)^{3} \mathrm{D}$ & 2.0 & $10959.30^{*}$ & $\left.\left.1\right|_{2} ^{1} \mathrm{D}\right)$ \\
\hline 1170.237 & $1.15(-6)$ & $1.60(1)$ & 2.0 & $10959.30^{*}$ & $\left.1 \mid{ }_{2}^{\mathrm{t}} \mathrm{D}\right)$ & 2.0 & $96412.10^{*}$ & $\left.\left.2\right|_{1} ^{2} \mathrm{D}\right)^{3} \mathrm{D}$ \\
\hline 1164.976 & $4.40(-6)$ & $6.39(0)$ & 2.0 & $10959.30^{*}$ & $\left.\left.1\right|_{2} ^{1} \mathrm{D}\right)$ & 3.0 & $96798.00^{*}$ & $\left.2 \mid{ }_{1}^{\frac{1}{2}} \mathrm{D}\right)^{3} \mathrm{D}$ \\
\hline 1152.839 & $1.03(-5)$ & $2.85(1)$ & 2.0 & $13458.30^{*}$ & $\left.\left.1\right|_{2} ^{3} \mathrm{P}\right)$ & 2.0 & $100200.70^{*}$ & $\left.\left.2\right|_{1} ^{2} \mathrm{D}\right)^{1} \mathrm{D}$ \\
\hline 1149.934 & $3.50(-6)$ & $3.12(-1)$ & 1.0 & $13239.20 *$ & $\left.\left.1\right|_{2} ^{3} \mathrm{P}\right)$ & 2.0 & $100200.70^{*}$ & $\left.\left.2\right|_{1} ^{2} \mathrm{D}\right)^{1} \mathrm{D}$ \\
\hline 1148.397 & - & $8.10(-1)$ & .0 & $13122.80^{*}$ & $\left.\left.1\right|_{2} ^{3} \mathrm{P}\right)$ & 2.0 & $100200.70^{*}$ & $\left.\left.2\right|_{1} ^{2} \mathrm{D}\right)^{1} \mathrm{D}$ \\
\hline 1120.556 & $1.53(-7)$ & $1.11(3)$ & 2.0 & $10959.30^{*}$ & $\left.\left.1\right|_{2} ^{1} \mathrm{D}\right)$ & 2.0 & $100200.70^{*}$ & $\left.2 \mid{ }_{1}^{2} \mathrm{D}\right)^{1} \mathrm{D}$ \\
\hline 1045.179 & - & $4.92(2)$ & 2.0 & $96412.10^{*}$ & $\left.\left.2\right|_{1} ^{2} \mathrm{D}\right)^{3} \mathrm{D}$ & 4.0 & $734.70^{*}$ & $\left.\left.1\right|_{2} ^{3} \mathrm{~F}\right)$ \\
\hline 1043.072 & - & $7.74(2)$ & 1.0 & $96196.10^{*}$ & $\left.\left.2\right|_{1} ^{2} \mathrm{D}\right)^{3} \mathrm{D}$ & 3.0 & $325.40^{*}$ & $\left.\left.1\right|_{2} ^{3} \mathrm{~F}\right)$ \\
\hline 1040.980 & $1.80(-9)$ & $1.79(3)$ & 3.0 & $96798.00^{*}$ & $\left.\left.2\right|_{1} ^{2} \mathrm{D}\right)^{3} \mathrm{D}$ & 4.0 & $734.70^{*}$ & $\left.1{ }_{2}^{3} \mathrm{~F}\right)$ \\
\hline 1040.727 & $8.19(-8)$ & $1.17(3)$ & 2.0 & $96412.10^{*}$ & $\left.\left.2\right|_{1} ^{2} \mathrm{D}\right)^{3} \mathrm{D}$ & 3.0 & $325.40^{*}$ & $\left.\left.1\right|_{2} ^{3} \mathrm{~F}\right)$ \\
\hline 1039.543 & $1.79(-8)$ & $1.57(3)$ & 1.0 & $96196.10^{*}$ & $\left.\left.2\right|_{1} ^{2} \mathrm{D}\right)^{3} \mathrm{D}$ & 2.0 & $.00^{*}$ & $\left.\left.1\right|_{2} ^{3} \mathrm{~F}\right)$ \\
\hline 1037.214 & $1.66(-8)$ & $6.83(2)$ & 2.0 & $.00 *$ & $\left.\left.1\right|_{2} ^{3} \mathrm{~F}\right)$ & 2.0 & $96412.10^{*}$ & $\left.\left.2\right|_{1} ^{2} \mathrm{D}\right)^{3} \mathrm{D}$ \\
\hline 1036.564 & $1.42(-9)$ & $5.13(2)$ & 3.0 & $325.40 *$ & $\left.\left.1\right|_{2} ^{3} \mathrm{~F}\right)$ & 3.0 & 96798.00* & $\left.\left.2\right|_{1} ^{\frac{2}{2}} \mathrm{D}\right)^{3} \mathrm{D}$ \\
\hline 1033.079 & $2.25(-9)$ & $4.94(1)$ & 2.0 & $.00^{*}$ & $\left.\left.1\right|_{2} ^{3} \mathrm{~F}\right)$ & 3.0 & 96798.00* & $\left.\left.2\right|_{1} ^{2} \mathrm{D}\right)^{3} \mathrm{D}$ \\
\hline 1005.369 & - & $1.52(0)$ & 2.0 & $100200.70^{*}$ & $\left.\left.2\right|_{1} ^{2} \mathrm{D}\right)^{1} \mathrm{D}$ & 4.0 & $734.70^{*}$ & $\left.\left.1\right|_{2} ^{3} \mathrm{~F}\right)$ \\
\hline 1001.249 & $1.22(-5)$ & $9.02(0)$ & 2.0 & $100200.70^{*}$ & $\left.\left.2\right|_{1} ^{2} \mathrm{D}\right){ }^{1} \mathrm{D}$ & 3.0 & $325.40^{*}$ & \\
\hline 997.997 & $6.19(-6)$ & $8.69(-1)$ & 2.0 & $.00 *$ & $\left.\left.1\right|_{2} ^{3} \mathrm{~F}\right)$ & 2.0 & $100200.70^{*}$ & $\left.\left.2\right|_{1} ^{2} \mathrm{D}\right)^{1} \mathrm{D}$ \\
\hline
\end{tabular}

